\title{
Fronteiras sul e sudeste: Uma análise morfométrica de pontas bifaciais de Minas Gerais, São Paulo, Paraná e Rio Grande do Sul (Brasil)
}

\author{
Mercedes Okumura ${ }^{1}$, Astolfo G. M. Araujo ${ }^{2}$ \\ 1. Programa de Pós-Graduação em Arqueologia, Departamento de Antropologia, Museu Nacional, Universidade \\ Federal do Rio de Janeiro, Brasil. E-mail: mercedes@mn.ufrj.br \\ 2. Instituto de Estudos Avançados e Museu de Arqueologia e Etnologia, Universidade de São Paulo, Brasil. \\ E-mail: astwolfo@usp.br
}

\section{Resumo:}

Estilo e função em artefatos arqueológicos ou etnográficos têm sido estudados por uma miríade de pesquisadores através das mais diversas abordagens teóricas. Nesse contexto, o estudo de pontas de projétil tem sido particularmente útil para gerar um maior conhecimento acerca desses artefatos como marcadores de identidade, refletindo potenciais fronteiras entre grupos. Este trabalho visa explorar as diferenças na morfologia de pontas bifaciais holocênicas oriundas de Minas Gerais, São Paulo, Paraná e Rio Grande do Sul à luz dos conceitos de estilo e função da Arqueologia Evolutiva. Foram analisadas 248 pontas através de morfometria geométrica em duas dimensões. Embora nossa análise envolva dados de morfometria geométrica da ponta completa, a forma dos pedúnculos foi considerada como sendo de particular importância. De acordo com as expectativas teóricas e os modelos heurísticos de estilo e função propostos por Dunnell (1978a), a especificidade na forma dos pedúnculos das pontas pode ser considerada um recurso estilístico cujo aspecto e replicação diferencial entre grupos se deu, muito provavelmente, por processos estocásticos. Assim, nossa expectativa teórica é que a forma dos pedúnculos reflita escolhas dos grupos e, em última instância, seja um reflexo de possíveis fronteiras culturais pré-históricas. Nossos resultados apontam para a presença de diferenças importantes no tamanho e forma geral das pontas de Minas Gerais, São Paulo e Paraná em relação às pontas do Rio Grande do Sul. Tais diferenças podem ser observadas também na morfologia dos pedúnculos, mostrando a utilidade da aplicação da dicotomia heurística entre estilo e função (sensu Dunnell 1978a) para melhor entender a presença de potenciais fronteiras culturais pretéritas.

Palavras-chave: Tradição Umbu; pontas líticas; estilo e função; morfometria geométrica; identidade

\section{Introdução: Pontas bifaciais como marcadores culturais}

Pontas bifaciais pré-históricas têm sido estudadas por arqueólogos sob diversos pontos de vista: tecnológico, experimental, morfológico e funcional, entre outros. Dentre todas essas abordagens, possivelmente o estudo da morfologia dessas pontas é o que mais tem sido

Published by the School of History, Classics and Archaeology, University of Edinburgh ISSN: 2055-0472. URL: http://journals.ed.ac.uk/lithicstudies/

This work is licensed under a Creative Commons Attribution 2.5 UK: Scotland License. 
explorado. A própria diversidade de tamanhos e formas observada no registro arqueológico seria um fator que permitiria e estimularia essa abordagem morfológica, ao mesmo tempo em que poderia servir para testar diversas hipóteses sobre a mudança (ou a falta dela) na forma dessas pontas, hipóteses estas relacionadas a tempo, função, tecnologia, fatores demográficos ou culturais (Bettinger \& Eerkens 1997). Assim, pontas bifaciais seriam veículos bastante adequados para carregar informações acerca de grupos e fronteiras devido à sua grande importância social, econômica, política e simbólica (Wiessner 1983).

Diversos estudos etnográficos têm dado suporte à hipótese de que a morfologia das pontas, assim como outros artefatos, poderia carregar informação acerca da identidade grupal. No caso dos Agta (um dos grupos de caçadores-coletores existente atualmente nas Filipinas), apesar do tipo de ponta utilizado estar relacionado às condições do ambiente e à presa, parece haver um estilo de ponta "onipresente" nessas comunidades, ou seja, é possível identificar esse grupo através das pontas manufaturadas por eles (Griffin 1997: 275). De fato, os Agta reconhecem que o estilo das pontas pode estar relacionado à identidade social do grupo (Griffin 1997: 281).

No caso dos San do deserto do Kalahari (Botswana), os membros dessas comunidades apontaram para a presença de diferenças entre os grupos, tanto em termos dos tipos de armas que eles preferiam usar, quanto no estilo ou formas das pontas (Hitchcock \& Bleed 1997). Quando perguntados sobre o porquê de manufaturar as pontas de um dado modo, os San respondiam que eles as faziam do modo como os seus ancestrais as faziam (Hitchcock \& Bleed 1997). Outro estudo feito por Wiessner (1983) com três grupos linguísticos San apontou para a presença de diferenças importantes reconhecidas tanto através da análise métrica e não métrica das pontas quanto através da simples observação de membros dos três grupos das pontas manufaturadas em cada grupo. Em termos práticos, essas diferenças no estilo das pontas serviriam, entre outros fatores, para a manutenção das fronteiras territoriais, uma vez que grupos não pertencentes a tais territórios poderiam ser identificados através das flechas encontradas junto a animais caçados por eles (Wiessner 1983).

Entre os pastoralistas africanos falantes de Maa, a idade e a posição dentro de seu grupo etário limitam a gama de lanças que um homem pode usar. Assim, o estilo das lanças auxilia e reflete a interação entre homens de diferentes status dentro do grupo. É interessante apontar que, nesse grupo, a maioria das inovações na morfologia das pontas resulta do contato com grupos vizinhos (Larick 1985).

Essas diferenças documentadas em grupos etnográficos estariam relacionadas ao chamado “estilo assertivo", definido como a variação formal na cultura material que é baseada no indivíduo e que carrega informações que apoiam a identidade individual através da diferenciação dos indivíduos semelhantes, assim como identificando os membros de um dado grupo (Wiessner 1984). No entanto, como Braun (1995: 137) nos alerta, o fato dos indivíduos serem capazes de discriminar artefatos de aparências distintas em uma dada escala física de proximidade, não significa que tais artefatos sempre terão um significado social. Ao fazer um paralelo entre estilo e marcadores étnicos, é possível listar três predições acerca da natureza da distribuição dos traços usados como marcadores e suas relações com grupos étnicos. A primeira predição refere-se ao fato das diferenças serem mais fortes nas áreas de fronteira do que dentro do território. A segunda predição diz que fronteiras de marcadores e de normas devem coincidir; a terceira predição diz que traços usados como marcadores que apresentam maiores diferenças potenciais são os mais marcantes (McElreath et al. 2003).

A ideia de que grupos diferentes produzem artefatos diferentes estaria por trás de diversos estudos que tentam inferir diferenças na morfologia das pontas. Essas diferenças podem estar relacionadas a aspectos temporais, funcionais ou estilísticos, embora seja bem aceito pelo menos desde Childe (1951) que as culturas arqueológicas (geralmente definidas em termos de estilo) não são necessariamente equivalentes a grupos étnicos (Shennan 1989). 
Ainda assim, alguns aspectos da morfologia das pontas poderiam ser utilizados como indicadores da existência de grupos humanos que compartilhavam um mesmo conceito de como uma ponta deveria ser feita. Estudos etnográficos apontam que artesãos são mais propensos a produzir seus produtos incorporando estilos semelhantes àqueles que compartilham suas identidades sociais. Assim, essa ideia proveniente da etnografia teria sido adotada por arqueólogos, como uma hipótese analítica (Braun 1995: 124). No entanto, o debate acerca da utilidade de tal ideia nunca foi muito produtivo, em parte porque não há uma concordância na definição dos termos “estilo” e "identidade social” entre arqueólogos; e em parte porque o registro etnográfico não pode prover a informação necessária para o andamento desse debate.

\subsection{Estilo e função: Uma confusão fundamental}

Muito já foi discutido a respeito da dicotomia estilo e função. Muitas vezes, arqueólogos aplicam o termo "variação estilística" a qualquer variação na aparência dos artefatos que não é ditada de forma absoluta por requerimentos mecânicos ou de manufatura (Braun, 1995: 124). Para Binford (1986) a variação estilística seria aquela que não poderia ser atribuída a outros fatores como material, função ou tecnologia. Para Conkey (1978), estilo seria um modo de transmitir informação e, portanto, estaria sujeito à seleção, podendo conferir uma vantagem adaptativa aos seus usuários. De acordo com Wiessner (1983), estilo pode ser definido como a variação formal da cultura material que transmite informação acerca da identidade pessoal e social. Seguindo a mesma linha, Wobst (1977) define estilo como a parte da variabilidade formal na cultura material que pode ser relacionada à participação dos artefatos nos processos de troca de informação, ou seja, o estilo seria importante no envio de mensagens entre indivíduos socialmente distantes (mas veja Hodder 1982: capítulo 3 para uma opinião diferente). Para esses autores, estilo seria uma forma de comunicar informação social, especialmente informação acerca da filiação de grupo. Para Braun \& Plog (1982), tal definição seria importante para estudos de mudança organizacional, uma vez que implica que mudanças no estilo ao longo do tempo refletiriam mudanças na estrutura das fronteiras sociais.

Para Roe (1995: 31), estilo é um sistema estruturado e intencional de seleção de certas dimensões de forma, processo ou princípio, função, significado e influência dentre possibilidades alternativas conhecidas para criar a variabilidade dentro de um corpus comportamental-artefatual. Para Hegmon (1992), estilo pode ser definido como um modo de fazer algo e que envolve uma escolha feita dentre várias alternativas. Da mesma forma, Sackett (1986: 630) propõe que as formas dos artefatos da cultura material representam escolhas particulares feitas pelos artesãos, consciente ou inconscientemente, de uma ampla gama de alternativas igualmente viáveis para se obter o mesmo fim. Tais escolhas constituem a chamada variação isocréstica. Hodder (1982: 205) enfatiza o contexto, dizendo que estilo é um modo particular na qual princípios gerais de significado são juntados e reorganizados em contextos locais como parte de estratégias sociais de indivíduos e grupos. Assim, haveria uma relação entre estrutura e função no estilo que não poderia ser examinada de forma adequada por abordagens linguísticas ou comportamentais (Hodder 1982: 207). Estilos poderiam expressar e justificar diferenças étnicas, porém, o modo na qual esses aspectos poderiam ser entendidos seria somente através do estudo das estruturas do significado simbólico (Hodder 1982: 205). Larick (1985) define estilo como o aspecto variável da cultura material que deriva de tradições sociais para a produção. Há ainda a definição de estilo tecnológico, na qual a tecnologia também seria considerada como um fenômeno cultural que seria expresso no estilo da própria tecnologia. Dessa forma, o estilo tecnológico seria o "pacote” definido pelas relações entre os muitos elementos que constituem as atividades tecnológicas. Esses 
elementos seriam unidos de forma não aleatória em um complexo de relações formais (Lecthman 1977: 6; Lechtman \& Steinberg 1979: 139; Childs 1991). A natureza não aleatória de estilo também é enfatizada por David \& Kramer (2001: 172), na qual estilo seria um potencial para interpretação residindo nas características formais de um artefato que são adquiridas no curso da manufatura como consequência do exercício de escolhas culturais. Essas escolhas não necessariamente são de natureza consciente; uma vez aprendidas, tornamse habituais e praticamente inconscientes (David \& Kramer 2001: 172). Os autores dão como exemplo o trabalho de Gosselain (1992: 572), na qual os ceramistas Bafia afirmam não existir alternativas possíveis às suas técnicas de dar forma aos vasos.

Dunnell (1978a), através da abordagem da Arqueologia Evolutiva, propõe que traços com valores seletivos discretos ao longo do tempo seriam sujeitos à seleção natural, ao passo que traços identificados como sendo neutros se comportariam de forma estocástica (ou seja, as condições atuais e futuras são relacionadas de forma probabilística - e não determinística às circunstâncias anteriores do ambiente seletivo, Hurt \& Rakita 2001: xxvi). Assim, para o autor, o estilo denotaria as formas que não apresentariam valores seletivos detectáveis, ao passo que a função seria manifestada pelas formas que afetariam de modo direto o fitness de uma população. Nessa discussão, torna-se importante não confundir função com propósito (O’Brien \& Leonard 2001: 3) ou com uso (Dunnell 1978b). Seguindo a mesma linha de Dunnell (1978a), Lipo et al. (1997; 2010) afirmam que o estilo seria a variação não limitada pela performance e Neiman (1995) define estilo como o aspecto não adaptativo da variação fenotípica, na qual o fitness seria o mesmo para toda a gama de estilos. Essas definições de estilo, também conhecidas como modelo neutro (contra Shennan \& Wilkinson 2001), implicam que uma grande variedade de escolhas seria essencialmente equivalente (Bentley 2011). Em contrapartida, Sackett (1985) propõe que o significado estilístico pode ser dado a qualquer atributo, seja este utilitário ou não. De fato, o autor chama a atenção para o fato de que quase todos os atributos que apresentam significado estilístico não são nem decorativos, nem particularmente elaborados. É provável que o autor não esteja usando a palavra “estilo” como ela foi definida por Dunnell (1978a), isto é, para denominar as variantes neutras da cultura material (Dunnell 2001: xix). Outra observação importante é que não se deve confundir as categorias usadas na observação com o fenômeno que está sendo observado. Desta forma, evita-se a noção errônea de que coisas são ou estilísticas ou funcionais (Dunnell 2001: xix). Assim, estilo como uma classe de fenômenos, certamente apresenta um custo e, portanto, estaria sob seleção. Em outras palavras, a quantidade de estilo não seria algo neutro. Porém, as diferenças de custo entre diferentes estilos seriam equivalentes, e, portanto, não estariam sob seleção (Dunnell 2001: xx).

Estudos apontam para a existência de "estilos" compartilhados em diferentes escalas de grupos humanos (Hegmon 1998: 265). Segundo Rick (1996), o foco no processo social exige a identificação de unidades estilísticas no registro arqueológico e torna-se, portanto, necessário reconhecer os diferentes níveis de escala: indivíduo, família, grupo local ou população regional. Para os arqueólogos que adotam as ideias de Dunnell, os indivíduos não agem ao acaso, porém, a análise estatística de suas escolhas, em termos populacionais, são comparáveis a cópias ao acaso (Bentley 2011).

A maioria dos estudos acerca do estilo em grupos presentes ou pretéritos não apresenta um mecanismo que explique a presença de diferentes estilos. A explicação de Dunnell (1978a) finalmente colocou o conceito de estilo de maneira formal, com implicações empíricas verificáveis, e deu aos arqueólogos a direção para construir métodos que são úteis para explorar porquê e como certos atributos culturais apresentam determinadas distribuições no tempo e no espaço (Lipo \& Madsen 2001: 94). De acordo com Neiman (1995), a dinâmica empírica é sempre considerada como sendo o resultado de intenções, crenças e desejos. No entanto, sob uma perspectiva evolutiva, a metáfora da personalidade é rejeitada em favor do 
entendimento de um sistema físico cuja dinâmica é causada por forças mecanicistas, não porque haja uma rejeição da ideia de intencionalidade em si, mas porque esse conceito é inócuo e, na verdade, não explica nada. Uma teoria consiste em descrições dos mecanismos e do modo que eles operam, seja em prosa ou em termos matemáticos. Nesse contexto, a Arqueologia Evolutiva, através da aplicação da Teoria Evolutiva a contextos arqueológicos, provê uma explicação teoricamente baseada de como e por que ocorre a continuidade e a mudança cultural ao longo das gerações.

No caso desta análise de pontas bifaciais, embora envolva dados de morfometria geométrica da ponta completa, será dada ênfase à morfologia dos pedúnculos. De acordo com as expectativas teóricas e os modelos heurísticos de estilo e função propostos por Dunnell (1978a), a forma diferencial dos pedúnculos das pontas pode ser considerada um recurso estilístico cuja aparência e adoção diferencial entre grupos foi, provavelmente, devido a processos estocásticos. Assim, nossa expectativa teórica é que a forma dos pedúnculos reflita escolhas dos grupos e, em última instância, seja um reflexo de possíveis fronteiras culturais pretéritas. Obviamente, a forma geral da ponta também pode se configurar como forte marcador cultural (por exemplo, pontas Clovis, Rabo-de-Peixe, etc.), desde que se configurem como alternativas equivalentes do ponto de vista funcional.

\subsection{O estudo das pontas bifaciais pré-históricas no Brasil}

A partir de meados dos anos 1960, arqueólogos brasileiros intensificaram seus trabalhos de reconhecimento do que seriam as principais tradições arqueológicas do país. No sul do país, o grande número de sítios arqueológicos que apresentava, geralmente, uma grande quantidade de pontas líticas lascadas bifacialmente teria sido determinante para a criação do termo Tradição Umbu (Miller 1967; 1974; Schmitz 1978: 108; Hilbert 1991: 16). Essa tradição se referia a sítios relacionados a grupos caçadores-coletores que apresentavam pontas bifaciais. Embora outros artefatos estivessem associados a essas pontas em diversos sítios, não estava claro quais outros artefatos seriam característicos dessa tradição ou que poderiam ser utilizados para descrevê-la. Dessa forma, ao longo do tempo, todos os sítios descobertos na região sul do país foram atribuídos à Tradição Umbu. Esse panorama tornou-se ainda mais complexo dada a falta de datações sistemáticas desses sítios. Estas, quando realizadas, revelaram idades que abrangiam todo o Holoceno e em alguns casos até mesmo o fim do Pleistoceno (Schmitz et al. 1980; Schmitz 1999; Noelli 2000, Okumura \& Araujo 2016). Outro fator complicador foi a descoberta de sítios que apresentavam pontas bifaciais nas demais regiões do país. Na região sudeste, em especial, os sítios com esse conteúdo acabaram sendo descritos como pertencentes à Tradição Umbu (Prous 1991: 154; Lima 2005; Koole 2007; 2014). No fim dos anos 1990, críticas severas ao uso indiscriminado desse termo se intensificaram e podem ser resumidas da seguinte forma (Dias 2003: 51; Dias 2007; Dias \& Hoeltz 2010; Hilbert 1994; Milder 1999): 1) definição dessa tradição foi baseada em poucos critérios tipológicos, 2) o uso do fóssil-guia (ou seja, a ponta bifacial) para determinar a associação de um dado sítio a essa tradição e 3) o uso de poucos atributos de classificação.

Juntamente com essas críticas, alguns pesquisadores procuraram entender a Tradição Umbu (e outras tradições arqueológicas, como a Humaitá) dentro de um contexto amplo regional e a partir de abordagens cujo foco foi principalmente a tecnologia de lascamento (Dias 2003; 2007; 2012; Dias \& Hoeltz 2010; entre outros). Outras abordagens, como a análise de restos faunísticos (Queiroz 2004; Rosa 2009) também foram importantes para um maior entendimento dessas tradições. Dentro desse panorama, acreditamos que a análise da morfologia das pontas bifaciais possa lançar luz sobre a definição e localização geográfica de possíveis fronteiras envolvendo caçadores-coletores do Holoceno nas regiões sudeste e sul brasileiras. Outros aspectos que podem ser explorados seriam as possíveis redes de troca de 
informação existentes entre esses grupos pretéritos, assim como as possíveis relações de ancestralidade e descendência entre esses grupos.

Nosso trabalho com as pontas bifaciais nos últimos anos teve por objetivo testar a hipótese de que há diferenças importantes na morfologia das pontas bifaciais do sudeste brasileiro em relação às do sul do Brasil, comumente associadas à Tradição Umbu. Essa hipótese foi testada a partir da aplicação da morfometria geométrica. Dessa forma, pretendeuse explorar a possibilidade de apontar a presença de possíveis grupos regionais e ou cronológicos a partir das diferenças morfológicas das pontas por eles utilizadas, especialmente no que diz respeito à parte estilística dessas. Nesse caso, assume-se que a forma do pedúnculo não estaria sujeita a fortes pressões seletivas em termos da performance das pontas de projétil, ou seja, poderia ser considerada como estilística de acordo com Dunnell (Okumura \& Araujo 2014).

\section{Métodos}

A morfometria geométrica é um método quantitativo de se estabelecer comparações de forma (Zelditch et al. 2004). Com o objetivo de resgatar a informação sobre a forma, quantificá-la e testar suas diferenças, incluiu-se uma análise utilizando a morfometria geométrica.

A morfometria quantifica e testa as diferenças na forma através do uso de pontos de referência (Bookstein 1991), também conhecidos na literatura internacional como "landmarks". Neste estudo, os pontos de referência utilizados foram cinco (indicados na Figura 1): o ápice da ponta (A), a ponta da aleta (B), o ponto de encontro entre pescoço e corpo da ponta (C), a lateral do pedúnculo (D) e a extremidade do pedúnculo na linha longitudinal (E). Esses cinco pontos de referência foram digitalizados com o software TPSDig2 (Rohlf 2017) em fotografias padronizadas (com a câmera paralela à ponta bifacial e com uma escala em cm) em 248 pontas de Minas Gerais, São Paulo, Paraná e Rio Grande do Sul (Tabela 1). Apenas exemplares que tivessem os cinco pontos de referência íntegros foram incluídos na amostra. A escolha dos sítios arqueológicos deu prioridade àqueles que apresentassem informação cronológica (Figura 2, Tabela 2). As análises de morfometria geométrica foram feitas com os softwares TPSRegr, TPSSmall, TPSRelw e TPSPLS (Rohlf 2017).

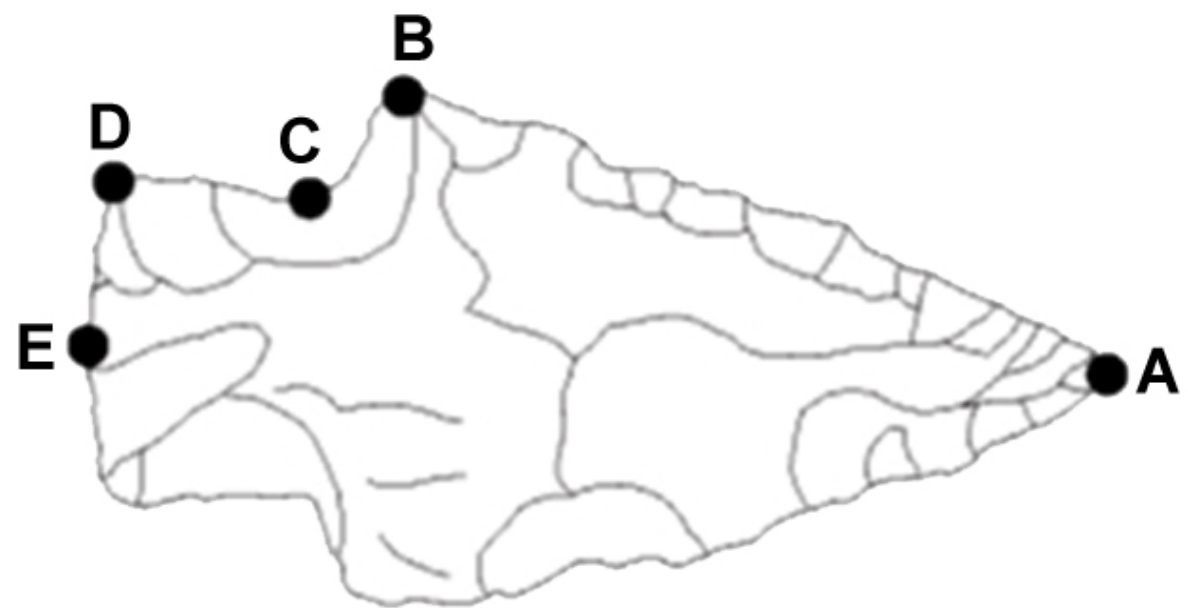

Figura 1. Ponta bifacial apresentando os pontos de referência das análises de morfometria geométrica. Figure 1. Bifacial point presenting geometric morphometrics analysis reference dots. 
Tabela 1. Amostra utilizada para análise de morfometria geométrica.

Table 1. Sample used for geometric morphometrics analysis.

\begin{tabular}{lllll}
\hline Estado & Sítio & Sigla & Município & $\#$ \\
\hline MG & Gruta do Marinheiro & Mar & Pimenta & 28 \\
SP & Alice Boer & Ali & Rio Claro & 37 \\
PR & PR-WB-16: Tunas & Tun & Arapoti & 14 \\
RS & RS-TQ-58: Garivaldino & Gar & Montenegro & 81 \\
RS & RS-C-61: Adelar Pilger & Pil & S. Sebastião do Caí & 5 \\
RS & RS-C-14: Bom Jardim Velho & Vel & S. Sebastião do Caí & 9 \\
RS & RS-217: Pedro Fridolino Schmitz & Sch & Bom Princípio & 6 \\
RS & RS-S-308: Morro da Flecha 1 & Fle & S. Francisco Paula & 7 \\
RS & RS-LN-01: Dalpiaz & Dal & Osório & 61 \\
\hline & Total & & $\mathbf{2 4 8}$ \\
\hline
\end{tabular}

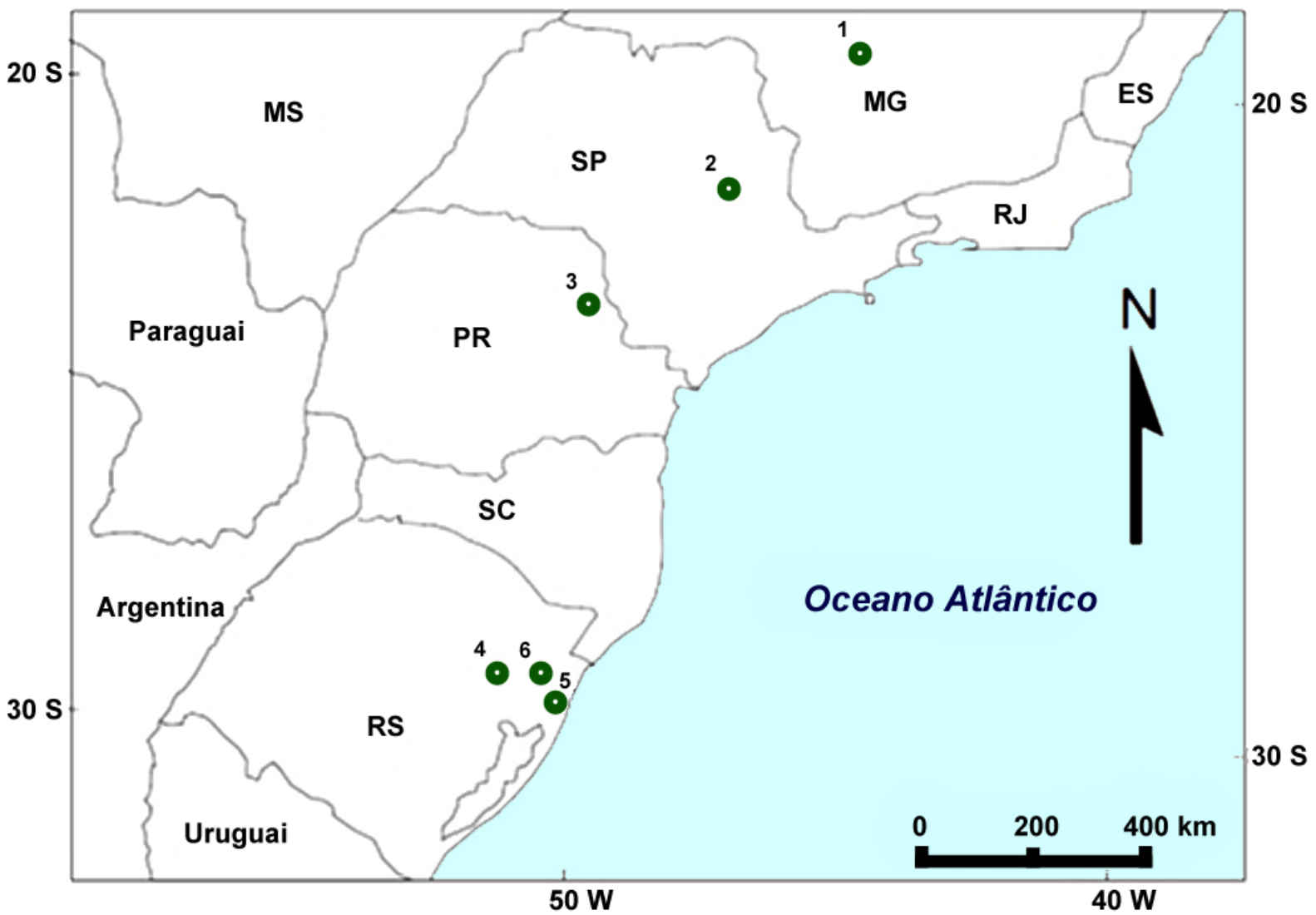

Figura 2. Mapa apresentando a localização dos sítios arqueológicos.

Figure 2. Map indicating the locations of archaeological sites.

1: Gruta do Marinheiro; 2: Alice Boer; 3: Tunas; 4: Garivaldino, A. Pilger, Bom Jd Velho, Pedro F. Schmitz; 5: Dalpiaz; 6: Morro da Flecha 1.

\section{Resultados}

Nas análises morfométricas, forma é definida como toda a informação geométrica que permanece depois que a localização, a escala e os efeitos de rotação são retirados de um dado objeto (Kendall 1977). O tamanho é frequentemente equivalente ao "tamanho do centróide", que é a raiz quadrada da soma das distâncias ao quadrado de todos os pontos de referência ao centro da forma. O tamanho do centróide é uma medida de tamanho que é matematicamente independente da forma (Zeldich et al. 2004: 13). 
Tabela 2. Sítios arqueológicos e seus respectivos estados de origem, datações absolutas (Anos AP) com o código do laboratório e datações calibradas (Anos Cal AP). As datações podem ser encontradas em Brochado \& Schmitz (1973); Miller (1974); Ribeiro et al. (1989); Ribeiro \& Ribeiro (1999); Schmitz (2006); Koole (2007; 2014); Chmyz et al. (2008); Araujo (2012); Dias (2012); Okumura \& Araujo (2014; 2015a). As datas foram calibradas com o software CalPal (Danzeglocke 2007) e a curva de calibração Intcal98 (Stuiver 2006).

Table 2. Archaeological sites and their respective states of origin in Brazil; absolute dates (years before present) with laboratory code; and calibrated dates (calibrated years before present). Dates can be found in Brochado \& Schmitz (1973); Miller (1974); Ribeiro et al. (1989); Ribeiro \& Ribeiro (1999); Schmitz (2006); Koole (2007; 2014); Chmyz et al. (2008); Araujo (2012); Dias (2012); Okumura \& Araujo (2014; 2015a, 2016). Dates were calibrated with the CalPal software (Danzeglocke 2007) and the Intcal98 calibration curve (Stuiver 2006).

\begin{tabular}{|c|c|c|c|}
\hline Estado & Sítio & Anos AP & Anos Cal AP \\
\hline \multirow[t]{2}{*}{ PR } & PR-WB-16: Tunas & $9630 \pm 40$ (Beta 210872) & $10980 \pm 140$ \\
\hline & & $7170 \pm 60($ Beta 210871$)$ & $7980 \pm 70$ \\
\hline \multirow[t]{3}{*}{ MG } & Gruta do Marinheiro & $9610 \pm 60($ Beta 230980$)$ & $10950 \pm 150$ \\
\hline & & $9460 \pm 40$ (Beta 337002) & $10780 \pm 160$ \\
\hline & & $9510 \pm 40$ (Beta 337003) & $10870 \pm 160$ \\
\hline \multirow[t]{4}{*}{ RS } & RS-TQ-58: Garivaldino & $9430 \pm 360$ (Beta 44739) & $10720 \pm 470$ \\
\hline & & $8290 \pm 130($ Beta 32183$)$ & $9260 \pm 170$ \\
\hline & & $8020 \pm 150($ Beta 33458) & $8920 \pm 240$ \\
\hline & & $7250 \pm 350($ Beta 44740) & $8090 \pm 330$ \\
\hline \multirow[t]{4}{*}{ RS } & RS-C-61: Adelar Pilger & $8430 \pm 50$ (Beta 260455) & $9440 \pm 60$ \\
\hline & & $8150 \pm 50($ Beta 260456$)$ & $9130 \pm 80$ \\
\hline & & $8030 \pm 50($ Beta 229583) & $8930 \pm 130$ \\
\hline & & $6180 \pm 50($ Beta 227856) & $7080 \pm 90$ \\
\hline \multirow[t]{2}{*}{ RS } & RS-217: Pedro Fridolino Schmitz & $7800 \pm 50$ (Beta 204345) & $8560 \pm 70$ \\
\hline & & $1400 \pm 40($ Beta 211727$)$ & $1320 \pm 30$ \\
\hline \multirow[t]{2}{*}{ SP } & Alice Boer & $7680 \pm 40$ (Beta 320199) & $8470 \pm 50$ \\
\hline & & $7200 \pm 40($ Beta 320198$)$ & $8020 \pm 60$ \\
\hline \multirow[t]{3}{*}{ RS } & RS-LN-01: Dalpiaz & $5950 \pm 190$ (SI 234) & $6800 \pm 240$ \\
\hline & & $5680 \pm 240($ SI 235) & $6490 \pm 270$ \\
\hline & & $4280 \pm 180$ (SI 233) & $4870 \pm 280$ \\
\hline RS & RS-C-14: Bom Jardim Velho & $5655 \pm 140$ (SI 1199) & $6470 \pm 150$ \\
\hline RS & RS-S-308: Morro da Flecha 1 & $575 \pm 80$ (SI 804) & $590 \pm 50$ \\
\hline
\end{tabular}

Foi aplicado o teste de Shapiro-Wilk para verificar a distribuição normal do tamanho do centróide em cada grupo. Foram retirados dois indivíduos do grupo Alice Boer e dois indivíduos do grupo Garivaldino a fim de garantir uma distribuição normal da variável. A Tabela 3 apresenta o resultado final do teste de Shapiro-Wilk e de Kolmogorov-Smimov. Ambos os testes apresentam resultados que permitem aceitar a hipótese de que a distribuição da variável é normal em cada grupo.

A Figura 3 apresenta os box-plots de cada grupo em relação ao tamanho do centróide.

A Análise de Variância (ANOVA) detectou a presença de diferenças significativas entre os grupos em relação ao tamanho do centróide (Tabela 4). Devido ao fato das variâncias serem homogêneas entre grupos $(p=0,60)$, foi possível aplicar os métodos de Tukey e de Bonferroni a fim de testar a diferença entre cada par de médias. As Tabelas 5 e 6 apresentam, respectivamente, os resultados das análises onde foram aplicados os métodos de Tukey e de Bonferroni. A Tabela 7 resume as diferenças encontradas nas duas análises. 
Tabela 3. Testes de normalidade de Shapiro-Wilk e de Kolmogorov-Smimov. Foram consideradas diferenças significativas ao nível de 0,05 (gl: graus de liberdade).

Table 3. Shapiro-Wilk and Kolmogorov-Smimov tests. Significant differences were considered at level 0.05 (gl: degrees of freedom).

\begin{tabular}{l|ccc|ccc}
\hline & \multicolumn{3}{|c|}{ Kolmogorov-Smirnov } & \multicolumn{3}{c}{ Shapiro-Wilk } \\
Grupo & Estatística & gl & Sig. & Estatística & gl & Sig. \\
\hline Mar & 0,148 & 28 & 0,117 & 0,932 & 28 & 0,070 \\
Ali & 0,126 & 35 & 0,171 & 0,945 & 35 & 0,079 \\
Tun & 0,178 & 14 & 0,200 & 0,909 & 14 & 0,153 \\
Gar & 0,052 & 79 & 0,200 & 0,991 & 79 & 0,846 \\
Pil & 0,331 & 5 & 0,078 & 0,805 & 5 & 0,089 \\
Vel & 0,209 & 9 & 0,200 & 0,905 & 9 & 0,283 \\
Sch & 0,293 & 6 & 0,118 & 0,857 & 6 & 0,180 \\
Fle & 0,187 & 7 & 0,200 & 0,929 & 7 & 0,541 \\
Dal & 0,097 & 61 & 0,200 & 0,961 & 61 & 0,048 \\
\hline
\end{tabular}

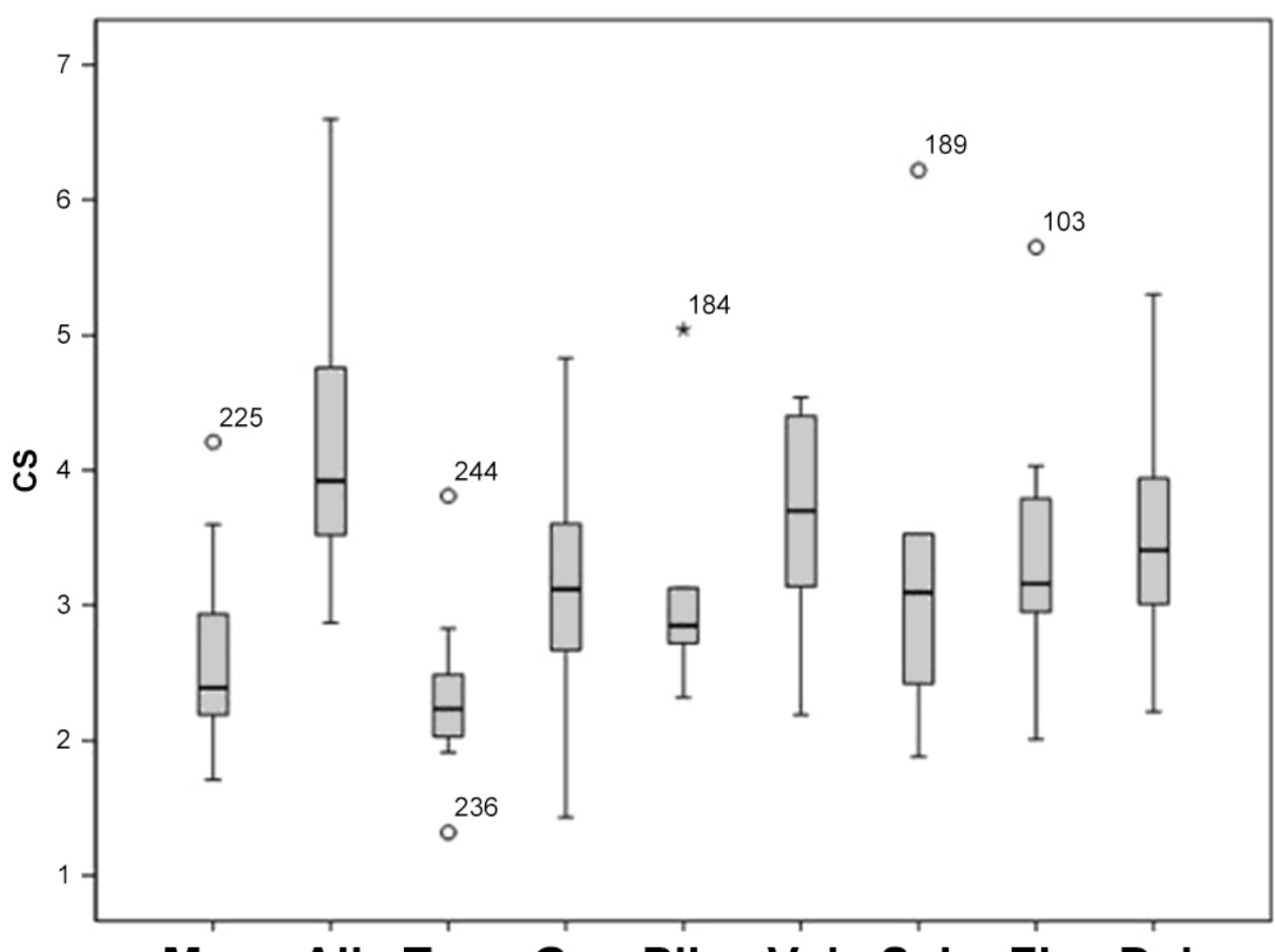

\section{Mar Ali Tun Gar Pil Vel Sch Fle Dal}

Figura 3. Box-plots referentes aos valores do tamanho do centróide de cada grupo.

Figure 3. Box-plots referring to the centroid size values of each group.

Tabela 4. Resultado da ANOVA para o tamanho do centróide de todos os grupos (gl: graus de liberdade, quadrado médio: divisão da soma dos quadrados pelos gl).

Table 4. ANOVA results for the centroid size of every group (gl: degrees of freedom, quadrado médio: division of the squares by $g l)$.

\begin{tabular}{lccccc}
\hline & Soma dos quadrados & gl & Quadrado médio & F & Sig. \\
\hline Entre grupos & 60,16 & 8 & 7,52 & 12,835 & 0 \\
Intra grupos & 137,688 & 235 & 0,586 & & \\
\hline Total & $\mathbf{1 9 7 , 8 4 9}$ & $\mathbf{2 4 3}$ & & & \\
\hline
\end{tabular}


Tabela 5. Resultados da aplicação do método de Tukey. * pares de médias onde diferenças significativas ao nível de 0,05 foram identificadas.

Table 5. Results of the application of the Tukey method. * pairs of means where significant differences at the level of 0.05 were identified.

\begin{tabular}{|c|c|c|c|c|c|c|}
\hline \multirow[b]{2}{*}{ (I) grupo } & \multirow[b]{2}{*}{ (J) grupo } & \multirow[b]{2}{*}{ Diferença das médias (I-J) } & \multirow[b]{2}{*}{ Erro padrão } & \multirow[b]{2}{*}{ Sig. } & \multicolumn{2}{|c|}{ 95\% intervalo de confiança } \\
\hline & & & & & Limite inferior & Limite superior \\
\hline \multirow[t]{8}{*}{ Mar } & Ali & $-1,5684285714 *$ & 0,194075837 & 0 & $-2,176070951$ & $-0,960786192$ \\
\hline & Tun & 0,233571429 & 0,250550828 & 0,991 & $-0,55089151$ & 1,018034367 \\
\hline & Gar & $-0,51261302$ & 0,16835017 & 0,064 & $-1,039709537$ & 0,014483497 \\
\hline & Pil & $-0,642714286$ & 0,371626934 & 0,728 & $-1,806260857$ & 0,520832286 \\
\hline & Vel & $-1,0418253968 *$ & 0,293301863 & 0,013 & $-1,96013983$ & $-0,123510963$ \\
\hline & Sch & $-0,804047619$ & 0,344349176 & 0,326 & $-1,882188804$ & 0,274093566 \\
\hline & Fle & $-0,902142857$ & 0,323459728 & 0,124 & $-1,914880156$ & 0,110594442 \\
\hline & Dal &,$- 9456323185^{*}$ & 0,174729124 & 0 & $-1,492701045$ & $-0,398563592$ \\
\hline \multirow[t]{8}{*}{ Ali } & Mar & $1,5684285714^{*}$ & 0,194075837 & 0 & 0,960786192 & 2,176070951 \\
\hline & Tun & $1,8020000000 *$ & 0,242055096 & 0 & 1,044136801 & 2,559863199 \\
\hline & Gar & $1,0558155515^{*}$ & 0,15542436 & 0 & 0,56918914 & 1,542441963 \\
\hline & Pil & 0,925714286 & 0,365952907 & 0,223 & $-0,220067173$ & 2,071495744 \\
\hline & Vel & 0,526603175 & 0,286078547 & 0,655 & $-0,369095394$ & 1,422301743 \\
\hline & Sch & 0,764380952 & 0,33821784 & 0,371 & $-0,294563306$ & 1,82332521 \\
\hline & Fle & 0,666285714 & 0,316924514 & 0,474 & $-0,325990136$ & 1,658561565 \\
\hline & Dal & $0,6227962529 *$ & 0,162312104 & 0,005 & 0,114604637 & 1,130987869 \\
\hline \multirow[t]{8}{*}{ Tun } & Mar & $-0,233571429$ & 0,250550828 & 0,991 & $-1,018034367$ & 0,55089151 \\
\hline & Ali & $-1,8020000000 *$ & 0,242055096 & 0 & $-2,559863199$ & $-1,044136801$ \\
\hline & Gar & $-0,7461844485^{*}$ & 0,221961751 & 0,025 & $-1,441136321$ & $-0,051232576$ \\
\hline & Pil & $-0,876285714$ & 0,398787935 & 0,411 & $-2,124872112$ & 0,372300683 \\
\hline & Vel & $-1,2753968254^{*}$ & 0,327033977 & 0,004 & $-2,299324931$ & $-0,251468719$ \\
\hline & Sch & $-1,037619048$ & 0,373499122 & 0,128 & $-2,207027352$ & 0,131789257 \\
\hline & Fle & $-1,1357142857 *$ & 0,354332379 & 0,04 & $-2,245112413$ & $-0,026316159$ \\
\hline & Dal & $-1,1792037471^{*}$ & 0,226838061 & 0 & $-1,889423121$ & $-0,468984373$ \\
\hline \multirow[t]{8}{*}{ Gar } & Mar & 0,51261302 & 0,16835017 & 0,064 & $-0,014483497$ & 1,039709537 \\
\hline & Ali & $-1,0558155515^{*}$ & 0,15542436 & 0 & $-1,542441963$ & $-0,56918914$ \\
\hline & Tun & $0,7461844485^{*}$ & 0,221961751 & 0,025 & 0,051232576 & 1,441136321 \\
\hline & Pil & $-0,130101266$ & 0,352984248 & 1 & $-1,235278459$ & 0,975075927 \\
\hline & Vel & $-0,529212377$ & 0,269290335 & 0,569 & $-1,372347836$ & 0,313923082 \\
\hline & Sch & $-0,291434599$ & 0,324141414 & 0,993 & $-1,306306226$ & 0,723437027 \\
\hline & Fle & $-0,389529837$ & 0,301856749 & 0,933 & $-1,334629218$ & 0,555569544 \\
\hline & Dal & $-0,4330192986 *$ & 0,130466732 & 0,029 & $-0,841504543$ & $-0,024534054$ \\
\hline \multirow[t]{8}{*}{ Pil } & Mar & 0,642714286 & 0,371626934 & 0,728 & $-0,520832286$ & 1,806260857 \\
\hline & Ali & $-0,925714286$ & 0,365952907 & 0,223 & $-2,071495744$ & 0,220067173 \\
\hline & Tun & 0,876285714 & 0,398787935 & 0,411 & $-0,372300683$ & 2,124872112 \\
\hline & Gar & 0,130101266 & 0,352984248 & 1 & $-0,975075927$ & 1,235278459 \\
\hline & Vel & $-0,399111111$ & 0,426945059 & 0,991 & $-1,735856146$ & 0,937633924 \\
\hline & Sch & $-0,161333333$ & 0,463500221 & 1 & $-1,612530875$ & 1,289864208 \\
\hline & Fle & $-0,259428571$ & 0,448198946 & 1 & $-1,662718537$ & 1,143861394 \\
\hline & Dal & $-0,302918033$ & 0,356070733 & 0,995 & $-1,417758865$ & 0,811922799 \\
\hline \multirow[t]{8}{*}{ Vel } & Mar & $1,0418253968 *$ & 0,293301863 & 0,013 & 0,123510963 & 1,96013983 \\
\hline & Ali & $-0,526603175$ & 0,286078547 & 0,655 & $-1,422301743$ & 0,369095394 \\
\hline & Tun & $1,2753968254^{*}$ & 0,327033977 & 0,004 & 0,251468719 & 2,299324931 \\
\hline & Gar & 0,529212377 & 0,269290335 & 0,569 & $-0,313923082$ & 1,372347836 \\
\hline & Pil & 0,399111111 & 0,426945059 & 0,991 & $-0,937633924$ & 1,735856146 \\
\hline & Sch & 0,237777778 & 0,40342516 & 1 & $-1,025327554$ & 1,50088311 \\
\hline & Fle & 0,13968254 & 0,385748235 & 1 & $-1,068077164$ & 1,347442243 \\
\hline & Dal & 0,096193078 & 0,273323566 & 1 & $-0,759570239$ & 0,951956396 \\
\hline
\end{tabular}




\begin{tabular}{|c|c|c|c|c|c|c|}
\hline \multirow[b]{2}{*}{ (I) grupo } & \multirow[b]{2}{*}{ (J) grupo } & \multirow[b]{2}{*}{ Diferença das médias (I-J) } & \multirow[b]{2}{*}{ Erro padrão } & \multirow[b]{2}{*}{ Sig. } & \multicolumn{2}{|c|}{ 95\% intervalo de confiança } \\
\hline & & & & & Limite inferior & Limite superior \\
\hline \multirow[t]{8}{*}{ Sch } & Mar & 0,804047619 & 0,344349176 & 0,326 & $-0,274093566$ & 1,882188804 \\
\hline & Ali & $-0,764380952$ & 0,33821784 & 0,371 & $-1,82332521$ & 0,294563306 \\
\hline & Tun & 1,037619048 & 0,373499122 & 0,128 & $-0,131789257$ & 2,207027352 \\
\hline & Gar & 0,291434599 & 0,324141414 & 0,993 & $-0,723437027$ & 1,306306226 \\
\hline & Pil & 0,161333333 & 0,463500221 & 1 & $-1,289864208$ & 1,612530875 \\
\hline & Vel & $-0,237777778$ & 0,40342516 & 1 & $-1,50088311$ & 1,025327554 \\
\hline & Fle & $-0,098095238$ & 0,42585452 & 1 & $-1,431425849$ & 1,235235372 \\
\hline & Dal & $-0,1415847$ & 0,327499838 & 1 & $-1,166971393$ & 0,883801994 \\
\hline \multirow[t]{8}{*}{ Fle } & Mar & 0,902142857 & 0,323459728 & 0,124 & $-0,110594442$ & 1,914880156 \\
\hline & Ali & $-0,666285714$ & 0,316924514 & 0,474 & $-1,658561565$ & 0,325990136 \\
\hline & Tun & $1,1357142857^{*}$ & 0,354332379 & 0,04 & 0,026316159 & 2,245112413 \\
\hline & Gar & 0,389529837 & 0,301856749 & 0,933 & $-0,555569544$ & 1,334629218 \\
\hline & Pil & 0,259428571 & 0,448198946 & 1 & $-1,143861394$ & 1,662718537 \\
\hline & Vel & $-0,13968254$ & 0,385748235 & 1 & $-1,347442243$ & 1,068077164 \\
\hline & Sch & 0,098095238 & 0,42585452 & 1 & $-1,235235372$ & 1,431425849 \\
\hline & Dal & $-0,043489461$ & 0,305460283 & 1 & $-0,999871338$ & 0,912892415 \\
\hline \multirow[t]{8}{*}{ Dal } & Mar & $0,9456323185^{*}$ & 0,174729124 & 0 & 0,398563592 & 1,492701045 \\
\hline & Ali & $-0,6227962529 *$ & 0,162312104 & 0,005 & $-1,130987869$ & $-0,114604637$ \\
\hline & Tun & 1,1792037471* & 0,226838061 & 0 & 0,468984373 & 1,889423121 \\
\hline & Gar & $0,4330192986 *$ & 0,130466732 & 0,029 & 0,024534054 & 0,841504543 \\
\hline & Pil & 0,302918033 & 0,356070733 & 0,995 & $-0,811922799$ & 1,417758865 \\
\hline & Vel & $-0,096193078$ & 0,273323566 & 1 & $-0,951956396$ & 0,759570239 \\
\hline & Sch & 0,1415847 & 0,327499838 & 1 & $-0,883801994$ & 1,166971393 \\
\hline & Fle & 0,043489461 & 0,305460283 & 1 & $-0,912892415$ & 0,999871338 \\
\hline
\end{tabular}


Tabela 6. Resultados da aplicação do método de Bonferroni. * pares de médias onde diferenças significativas ao nível de 0,05 foram identificadas.

Table 6. Results of the application of the Bonferroni method. * pairs of means where significant differences at the level of 0.05 level were identified.

\begin{tabular}{|c|c|c|c|c|c|c|}
\hline \multirow[b]{2}{*}{ (I) grupo } & \multirow[b]{2}{*}{ (J) grupo } & \multirow[b]{2}{*}{ Diferença das médias (I-J) } & \multirow[b]{2}{*}{ Erro padrão } & \multirow[b]{2}{*}{ Sig. } & \multicolumn{2}{|c|}{ 95\% intervalo de confiança } \\
\hline & & & & & Limite inferior & Limite superior \\
\hline \multirow[t]{8}{*}{ Mar } & Ali & $-1,5684285714 *$ & 0,194075837 & 0 & $-2,196366775$ & $-0,940490368$ \\
\hline & Tun & 0,233571429 & 0,250550828 & 1 & $-0,577093307$ & 1,044236164 \\
\hline & Gar & $-0,51261302$ & 0,16835017 & 0,093 & $-1,057315054$ & 0,032089014 \\
\hline & Pil & $-0,642714286$ & 0,371626934 & 1 & $-1,845124402$ & 0,559695831 \\
\hline & Vel & $-1,0418253968 *$ & 0,293301863 & 0,017 & $-1,990812392$ & $-0,092838401$ \\
\hline & Sch & $-0,804047619$ & 0,344349176 & 0,734 & $-1,918199729$ & 0,310104491 \\
\hline & Fle & $-0,902142857$ & 0,323459728 & 0,206 & $-1,94870653$ & 0,144420816 \\
\hline & Dal &,$- 9456323185^{*}$ & 0,174729124 & 0 & $-1,510973653$ & $-0,380290984$ \\
\hline \multirow[t]{8}{*}{ Ali } & Mar & $1,5684285714^{*}$ & 0,194075837 & 0 & 0,940490368 & 2,196366775 \\
\hline & Tun & $1,8020000000 *$ & 0,242055096 & 0 & 1,01882346 & 2,58517654 \\
\hline & Gar & $1,0558155515^{*}$ & 0,15542436 & 0 & 0,552935362 & 1,558695741 \\
\hline & Pil & 0,925714286 & 0,365952907 & 0,435 & $-0,258337347$ & 2,109765918 \\
\hline & Vel & 0,526603175 & 0,286078547 & 1 & $-0,399012565$ & 1,452218914 \\
\hline & Sch & 0,764380952 & 0,33821784 & 0,89 & $-0,329933035$ & 1,85869494 \\
\hline & Fle & 0,666285714 & 0,316924514 & 1 & $-0,359133079$ & 1,691704507 \\
\hline & Dal & ,6227962529* & 0,162312104 & 0,006 & 0,097630561 & 1,147961944 \\
\hline \multirow[t]{8}{*}{ Tun } & Mar & $-0,233571429$ & 0,250550828 & 1 & $-1,044236164$ & 0,577093307 \\
\hline & Ali & $-1,8020000000 *$ & 0,242055096 & 0 & $-2,58517654$ & $-1,01882346$ \\
\hline & Gar & $-0,7461844485^{*}$ & 0,221961751 & 0,033 & $-1,464348364$ & $-0,028020532$ \\
\hline & Pil & $-0,876285714$ & 0,398787935 & 1 & $-2,166576067$ & 0,414004638 \\
\hline & Vel & $-1,2753968254^{*}$ & 0,327033977 & 0,005 & $-2,333525089$ & $-0,217268562$ \\
\hline & Sch & $-1,037619048$ & 0,373499122 & 0,213 & $-2,246086684$ & 0,170848589 \\
\hline & Fle & $-1,135714286$ & 0,354332379 & 0,055 & $-2,282167349$ & 0,010738777 \\
\hline & Dal & $-1,1792037471^{*}$ & 0,226838061 & 0 & $-1,913145113$ & $-0,445262381$ \\
\hline \multirow[t]{8}{*}{ Gar } & Mar & 0,51261302 & 0,16835017 & 0,093 & $-0,032089014$ & 1,057315054 \\
\hline & Ali & $-1,0558155515^{*}$ & 0,15542436 & 0 & $-1,558695741$ & $-0,552935362$ \\
\hline & Tun & $0,7461844485^{*}$ & 0,221961751 & 0,033 & 0,028020532 & 1,464348364 \\
\hline & Pil & $-0,130101266$ & 0,352984248 & 1 & $-1,272192412$ & 1,01198988 \\
\hline & Vel & $-0,529212377$ & 0,269290335 & 1 & $-1,40050935$ & 0,342084596 \\
\hline & Sch & $-0,291434599$ & 0,324141414 & 1 & $-1,340203888$ & 0,75733469 \\
\hline & Fle & $-0,389529837$ & 0,301856749 & 1 & $-1,366196423$ & 0,587136748 \\
\hline & Dal & $-0,4330192986 *$ & 0,130466732 & 0,038 & $-0,855148333$ & $-0,010890264$ \\
\hline \multirow[t]{8}{*}{ Pil } & Mar & 0,642714286 & 0,371626934 & 1 & $-0,559695831$ & 1,845124402 \\
\hline & Ali & $-0,925714286$ & 0,365952907 & 0,435 & $-2,109765918$ & 0,258337347 \\
\hline & Tun & 0,876285714 & 0,398787935 & 1 & $-0,414004638$ & 2,166576067 \\
\hline & Gar & 0,130101266 & 0,352984248 & 1 & $-1,01198988$ & 1,272192412 \\
\hline & Vel & $-0,399111111$ & 0,426945059 & 1 & $-1,780504682$ & 0,98228246 \\
\hline & Sch & $-0,161333333$ & 0,463500221 & 1 & $-1,661002232$ & 1,338335565 \\
\hline & Fle & $-0,259428571$ & 0,448198946 & 1 & $-1,709589735$ & 1,190732593 \\
\hline & Dal & $-0,302918033$ & 0,356070733 & 1 & $-1,454995593$ & 0,849159527 \\
\hline \multirow[t]{8}{*}{ Vel } & Mar & $1,0418253968 *$ & 0,293301863 & 0,017 & 0,092838401 & 1,990812392 \\
\hline & Ali & $-0,526603175$ & 0,286078547 & 1 & $-1,452218914$ & 0,399012565 \\
\hline & Tun & $1,2753968254^{*}$ & 0,327033977 & 0,005 & 0,217268562 & 2,333525089 \\
\hline & Gar & 0,529212377 & 0,269290335 & 1 & $-0,342084596$ & 1,40050935 \\
\hline & Pil & 0,399111111 & 0,426945059 & 1 & $-0,98228246$ & 1,780504682 \\
\hline & Sch & 0,237777778 & 0,40342516 & 1 & $-1,067516455$ & 1,54307201 \\
\hline & Fle & 0,13968254 & 0,385748235 & 1 & $-1,108417469$ & 1,387782548 \\
\hline & Dal & 0,096193078 & 0,273323566 & 1 & $-0,788153536$ & 0,980539692 \\
\hline
\end{tabular}




\begin{tabular}{lllllll}
\hline \multirow{2}{*}{ (I) grupo } & (J) grupo & Diferença das médias (I-J) & Erro padrão & Sig. & Limite inferior & Limite superior \\
\hline Sch & Mar & 0,804047619 & 0,344349176 & 0,734 & $-0,310104491$ & 1,918199729 \\
& Ali & $-0,764380952$ & 0,33821784 & 0,89 & $-1,85869494$ & 0,329933035 \\
& Tun & 1,037619048 & 0,373499122 & 0,213 & $-0,170848589$ & 2,246086684 \\
& Gar & 0,291434599 & 0,324141414 & 1 & $-0,75733469$ & 1,340203888 \\
& Pil & 0,161333333 & 0,463500221 & 1 & $-1,338335565$ & 1,661002232 \\
& Vel & $-0,237777778$ & 0,40342516 & 1 & $-1,54307201$ & 1,067516455 \\
& Fle & $-0,098095238$ & 0,42585452 & 1 & $-1,47596034$ & 1,279769863 \\
& Dal & $-0,1415847$ & 0,327499838 & 1 & $-1,201220269$ & 0,91805087 \\
\hline Fle & Mar & 0,902142857 & 0,323459728 & 0,206 & $-0,144420816$ & 1,94870653 \\
& Ali & $-0,666285714$ & 0,316924514 & 1 & $-1,691704507$ & 0,359133079 \\
& Tun & 1,135714286 & 0,354332379 & 0,055 & $-0,010738777$ & 2,282167349 \\
& Gar & 0,389529837 & 0,301856749 & 1 & $-0,587136748$ & 1,366196423 \\
& Pil & 0,259428571 & 0,448198946 & 1 & $-1,190732593$ & 1,709589735 \\
& Vel & $-0,13968254$ & 0,385748235 & 1 & $-1,387782548$ & 1,108417469 \\
& Sch & 0,098095238 & 0,42585452 & 1 & $-1,279769863$ & 1,47596034 \\
& Dal & $-0,043489461$ & 0,305460283 & 1 & $-1,031815388$ & 0,944836465 \\
\hline Dal & Mar & $0,9456323185^{*}$ & 0,174729124 & 0 & 0,380290984 & 1,510973653 \\
& Ali & $-0,6227962529 *$ & 0,162312104 & 0,006 & $-1,147961944$ & $-0,097630561$ \\
& Tun & $1,1792037471^{*}$ & 0,226838061 & 0 & 0,445262381 & 1,913145113 \\
& Gar & $0,4330192986 *$ & 0,130466732 & 0,038 & 0,010890264 & 0,855148333 \\
& Pil & 0,302918033 & 0,356070733 & 1 & $-0,849159527$ & 1,454995593 \\
& Vel & $-0,096193078$ & 0,273323566 & 1 & $-0,980539692$ & 0,788153536 \\
& Sch & 0,1415847 & 0,327499838 & 1 & $-0,91805087$ & 1,201220269 \\
& Fle & 0,043489461 & 0,305460283 & 1 & $-0,944836465$ & 1,031815388 \\
\hline
\end{tabular}

Tabela 7. Resumo das diferenças encontradas na aplicação dos métodos de Tukey e de Bonferroni. As letras T e B indicam, respectivamente, onde foram observadas diferenças significativas entre os grupos utilizando-se o método de Tukey e de Bonferroni.

Table 7. Summary of differences found in the application of the Tukey and Bonferroni methods. The letters $\mathrm{T}$ and B indicate, respectively, where significant differences were found between groups using the Tukey and Bonferroni methods.

\begin{tabular}{lcccccccc}
\hline & Mar & Ali & Tun & Gar & Pil & Vel & Sch & Fle \\
\hline Ali & TB & & & & & & & \\
Tun & & TB & & & & & & \\
Gar & & TB & TB & & & & & \\
Pil & & & & & & & & \\
Vel & TB & & TB & & & & & \\
Sch & & & & & & & & \\
Fle & & & T & & & & & \\
Dal & TB & TB & TB & TB & & & & \\
\hline
\end{tabular}

Através da análise da Figura 3 e das Tabelas 5 a 7, é possível verificar que as pontas dos sítios Alice Boer, Bom Jardim Velho e Dalpiaz são significativamente diferentes das pontas da Gruta do Marinheiro (MG). Essa diferença se dá em relação ao tamanho maior das pontas desses grupos em relação às pontas de Minas Gerais. As pontas dos grupos Garivaldino, Bom Jardim Velho, Dalpiaz e Morro da Flecha 1 (neste último caso, somente na análise de Tukey) são maiores em comparação aos materiais de Tunas, da mesma forma que as pontas de Dalpiaz são maiores que as de Garivaldino. Já as pontas dos grupos Tunas, Garivaldino e Dalpiaz são significativamente menores em comparação às pontas de Alice Boer. 


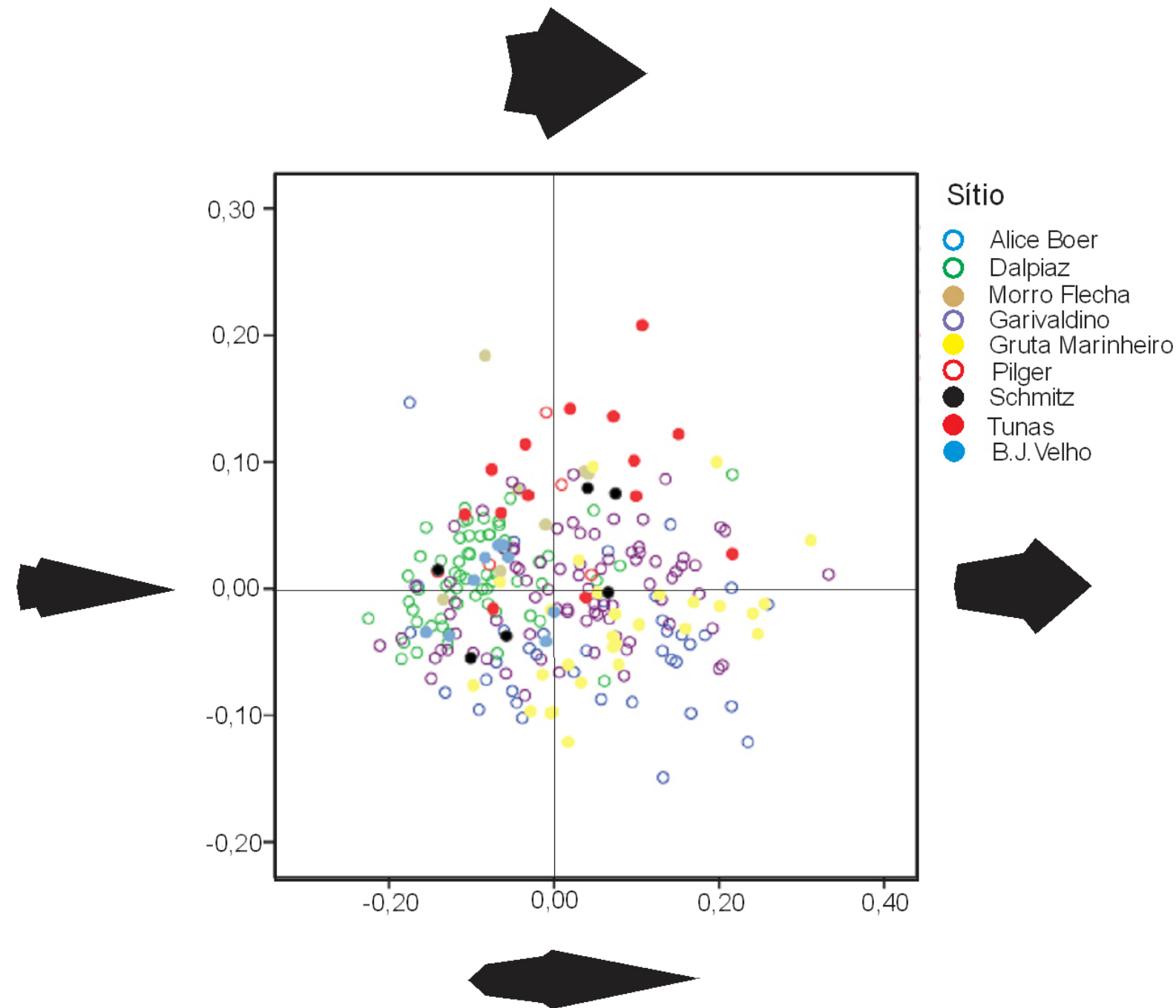

Figura 4. Gráfico de Análise de Componentes Principais das coordenadas da forma. As formas extremas de cada eixo são apresentadas.

Figure 4. Principal Components Analysis graph of the shape coordinates. The extreme shapes of each axis are presented.

A Figura 4 apresenta o gráfico de ordenação das deformações relativas (em inglês chamado "Relative Warps Ordination Plot", que nada mais é do que uma Análise de Componentes Principais das coordenadas da forma), onde os espécimes são distribuídos de acordo com a sua forma (portanto, sem a informação de tamanho, que já foi analisada). No centro do gráfico encontra-se a forma consenso, ou seja, a forma média da amostra analisada. No lado direito do gráfico é possível observar que as pontas foram provavelmente reavivadas, apresentando o corpo muito curto em relação ao pedúnculo. Os espécimes de Dalpiaz concentram-se nessa parte do gráfico. No lado esquerdo, ocorre o padrão oposto. No eixo vertical, podem observar-se as diferenças entre os pedúnculos das pontas: na parte superior, as bases são côncavas, enquanto na parte inferior, as bases são convexas ou afiladas. Na parte superior direita do gráfico localizam-se a maior parte das pontas do Tunas, ao passo que na parte inferior direita do gráfico encontram-se as pontas da Gruta do Marinheiro. Ou seja, esses dois grupos apresentam diferenças em termos da forma da base do pedúnculo, porém apresentam em comum alta frequência de reavivamento do corpo das pontas. As pontas de Dalpiaz encontram-se majoritariamente na porção esquerda (tanto superior quanto inferior) do gráfico, indicando a raridade do processo de reavivamento desses materiais. As pontas de Alice Boer encontram-se tanto na parte esquerda, quanto na parte direita inferior do gráfico, 
sendo caracterizadas por bases do pedúnculo convexas ou afiladas. Os espécimes de Garivaldino parecem abranger toda a diversidade morfológica apresentada no gráfico, não mostrando uma clara concentração em nenhum quadrante (Okumura \& Araujo 2014). Os demais grupos, por apresentarem tamanho amostral pequeno, não mostram uma tendência clara na distribuição dos espécimes no gráfico.

Análises de Funções Discriminantes com base na matriz de "Relative Warp Scores" foram realizadas a fim de verificar se haveria uma alta porcentagem de classificação correta, indicando distinção entre os grupos. A Figura 5 apresenta o gráfico da primeira e segunda função discriminante, onde é possível ver que há diferenças nítidas entre alguns grupos, especialmente Gruta do Marinheiro, Alice Boer, Tunas e Morro da Flecha 1. Todos esses grupos, à exceção de Morro da Flecha 1, apresentam tamanho amostral razoável. É possível verificar que os grupos de tamanho amostral pequeno (Adelar Pilger, Bom Jardim Velho, Pedro Fridolino Schmitz, todos do Rio Grande do Sul) se concentram próximos a Dalpiaz. Os materiais de Garivaldino, por sua vez, encontram-se próximos a essa concentração de sítios do sul. A alta porcentagem de classificação correta verificada em todos os grupos de tamanho amostral grande sugere a presença de diferenças importantes na forma dos mesmos.

\section{Canonical Discriminant Functions}

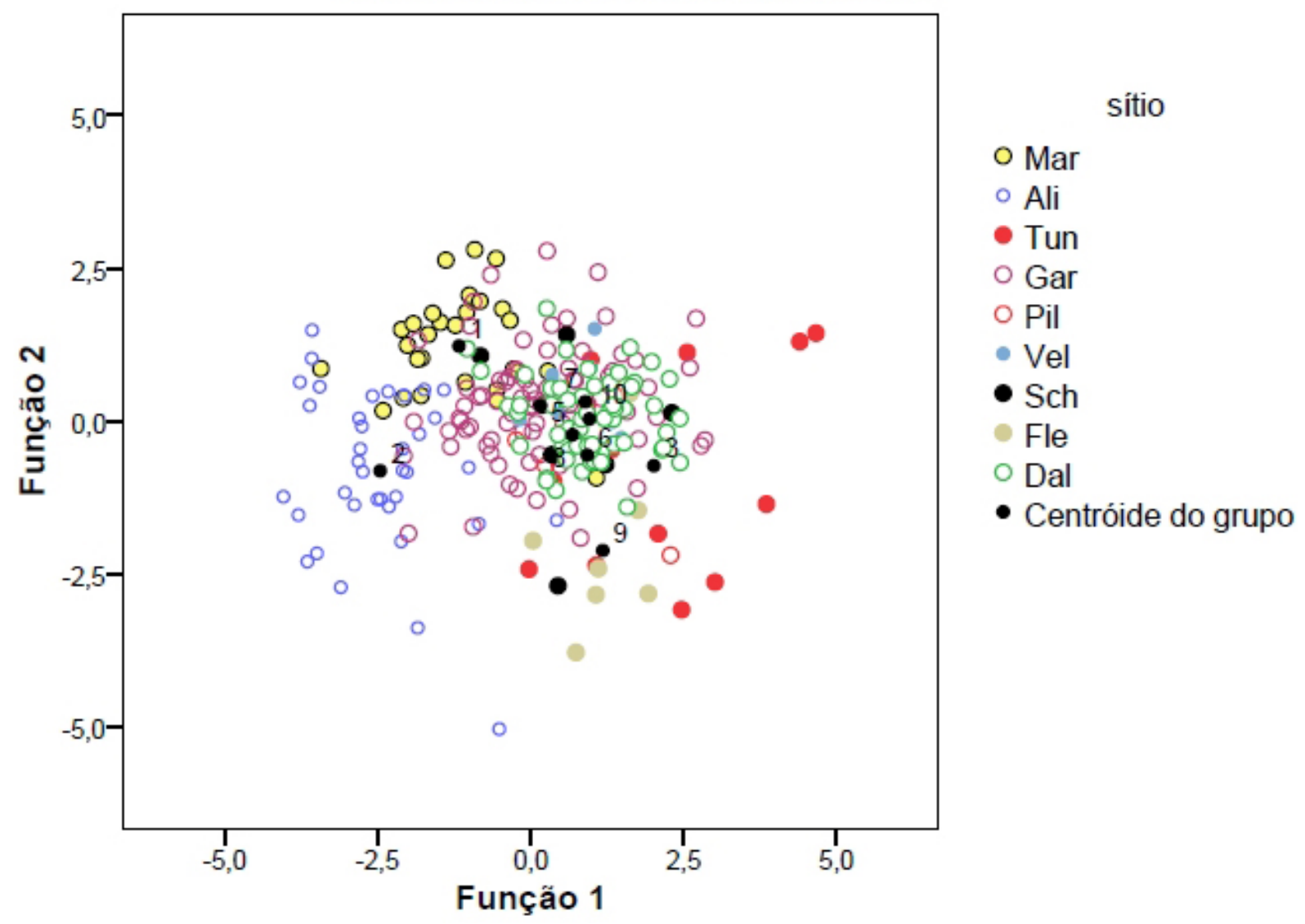

Figura 5. Gráfico da Função 1 x Função 2 da Análise de Funções Discriminantes com base na matriz de "Relative Warp Scores".

Figure 5. Function 1 x Function 2 Discriminant Analysis Functions Analysis graph based on the "Relative Warp Scores" matrix.

A classificação geral correta de 70,6\% (Tabela 8) a 65,7\% (Tabela 9) também sugere que os grupos em geral apresentam formas distintas. Essa divergência na porcentagem de classificação correta deve-se ao critério de incluir ou excluir o indivíduo a ser classificado da amostra que gerará a função discriminante. A Tabela 8 apresenta os resultados quando todos 
os indivíduos, inclusive aquele a ser classificado, são incluídos no cálculo. A Tabela 9 apresenta a classificação quando o indivíduo a ser classificado não faz parte do cálculo (em inglês, esta análise chama-se "cross-validated", que poderia ser traduzido por "validação cruzada”). Os resultados apresentados na Tabela 8 ajudam a entender os resultados da Figura 5, uma vez que os grupos de tamanho amostral pequeno (Adelar Pilger, Bom Jardim Velho e Pedro Fridolino Schmitz) observados próximos a Dalpiaz na figura apresentam muitos espécimes classificados de forma errônea como pertencendo a Dalpiaz.

Tabela 8. Resultados de classificação da Análise de Funções Discriminantes.

Table 8. Results of the classification of Discriminant Functions Analysis.

\begin{tabular}{cccccccccccc}
\hline & & Mar & Ali & Tun & Gar & Pil & Vel & Sch & Fle & Dal & Total \\
\hline Número & Mar & 17 & 2 & 1 & 8 & 0 & 0 & 0 & 0 & 0 & $\mathbf{2 8}$ \\
& Ali & 1 & 32 & 0 & 2 & 0 & 0 & 0 & 1 & 1 & $\mathbf{3 7}$ \\
& Tun & 0 & 0 & 11 & 1 & 0 & 0 & 0 & 0 & 2 & $\mathbf{1 4}$ \\
& Gar & 4 & 3 & 1 & 60 & 0 & 0 & 0 & 0 & 13 & $\mathbf{8 1}$ \\
& Pil & 0 & 0 & 0 & 1 & 1 & 0 & 0 & 0 & 3 & $\mathbf{5}$ \\
& Vel & 0 & 0 & 0 & 3 & 0 & 0 & 0 & 0 & 6 & $\mathbf{9}$ \\
& Sch & 1 & 0 & 0 & 2 & 0 & 0 & 0 & 1 & 2 & $\mathbf{6}$ \\
& Fle & 0 & 0 & 0 & 0 & 0 & 0 & 0 & 5 & 2 & $\mathbf{7}$ \\
& Dal & 0 & 0 & 0 & 12 & 0 & 0 & 0 & 0 & 49 & $\mathbf{6 1}$ \\
\hline \% & Mar & 60,7 & 7,1 & 3,6 & 28,6 & 0 & 0 & 0 & 0 & 0 & $\mathbf{1 0 0}$ \\
& Ali & 2,7 & 86,5 & 0 & 5,4 & 0 & 0 & 0 & 2,7 & 2,7 & $\mathbf{1 0 0}$ \\
& Tun & 0 & 0 & 78,6 & 7,1 & 0 & 0 & 0 & 0 & 14,3 & $\mathbf{1 0 0}$ \\
& Gar & 4,9 & 3,7 & 1,2 & 74,1 & 0 & 0 & 0 & 0 & 16,0 & $\mathbf{1 0 0}$ \\
& Pil & 0 & 0 & 0 & 20,0 & 20,0 & 0 & 0 & 0 & 60,0 & $\mathbf{1 0 0}$ \\
& Vel & 0 & 0 & 0 & 33,3 & 0 & 0 & 0 & 0 & 66,7 & $\mathbf{1 0 0}$ \\
& Sch & 16,7 & 0 & 0 & 33,3 & 0 & 0 & 0 & 16,7 & 33,3 & $\mathbf{1 0 0}$ \\
& Fle & 0 & 0 & 0 & 0 & 0 & 0 & 0 & 71,4 & 28,6 & $\mathbf{1 0 0}$ \\
& Dal & 0 & 0 & 0 & 19,7 & 0 & 0 & 0 & 0 & 80,3 & $\mathbf{1 0 0}$ \\
\hline
\end{tabular}

A partir dos "quadrados mínimos parciais", foi testada a correlação entre "grupo" e "forma". A correlação entre grupo e forma variou de 0,59840 a 0,34578, indicando uma relação moderada ao menos entre o primeiro vetor da forma e os grupos. Essa relação é apoiada pelos resultados observados nas análises anteriores, onde diferenças importantes foram verificadas entre as formas dos diversos grupos. A correlação entre tamanho e forma $(0,49739)$ indicou uma alometria mediana nesta amostra, ou seja, mudanças no tamanho das pontas não implicam fortemente em mudanças na forma e vice-versa. Esse resultado se dá, em parte, devido à relativamente grande variação de tamanho e forma observada na amostra, o que faz com que pontas de um mesmo tamanho não apresentem homogeneidade na forma (por exemplo, embora as pontas da Gruta do Marinheiro e de Tunas sejam de tamanho pequeno, apresentam formas muito distintas).

\section{Discussão e conclusões}

Nossos resultados apontam para uma clara diferenciação na morfologia das pontas provenientes da região sudeste (Gruta do Marinheiro e Alice Boer) e do Paraná (Tunas) em comparação aos materiais do Rio Grande do Sul, que parecem ser mais homogêneos. Apesar desse resultado, algumas diferenças importantes foram observadas nas pontas de Dalpiaz e Morro da Flecha 1. 
Tabela 9. Resultados de classificação da Análise de Funções Discriminantes (“validação cruzada”).

Table 9. Results of the classification of Discriminant Functions Analysis ("cross-validation”).

\begin{tabular}{cccccccccccc}
\hline & & Mar & Ali & Tun & Gar & Pil & Vel & Sch & Fle & Dal & Total \\
\hline Número & Mar & 15 & 2 & 1 & 9 & 0 & 0 & 0 & 0 & 1 & $\mathbf{2 8}$ \\
& Ali & 2 & 30 & 0 & 2 & 0 & 0 & 0 & 1 & 2 & $\mathbf{3 7}$ \\
& Tun & 0 & 0 & 9 & 2 & 0 & 0 & 0 & 1 & 2 & $\mathbf{1 4}$ \\
& Gar & 5 & 4 & 1 & 56 & 0 & 0 & 0 & 0 & 15 & $\mathbf{8 1}$ \\
& Pil & 0 & 0 & 1 & 1 & 0 & 0 & 0 & 0 & 3 & $\mathbf{5}$ \\
& Vel & 0 & 0 & 0 & 3 & 0 & 0 & 0 & 0 & 6 & $\mathbf{9}$ \\
& Sch & 1 & 0 & 0 & 2 & 0 & 0 & 0 & 1 & 2 & $\mathbf{6}$ \\
& Fle & 0 & 0 & 0 & 0 & 0 & 0 & 0 & 4 & 3 & $\mathbf{7}$ \\
& Dal & 0 & 0 & 0 & 12 & 0 & 0 & 0 & 0 & 49 & $\mathbf{6 1}$ \\
\hline & Mar & 53,6 & 7,1 & 3,6 & 32,1 & 0 & 0 & 0 & 0 & 3,6 & $\mathbf{1 0 0}$ \\
& Ali & 5,4 & 81,1 & 0 & 5,4 & 0 & 0 & 0 & 2,7 & 5,4 & $\mathbf{1 0 0}$ \\
& Tun & 0 & 0 & 64,3 & 14,3 & 0 & 0 & 0 & 7,1 & 14,3 & $\mathbf{1 0 0}$ \\
& Gar & 6,2 & 4,9 & 1,2 & 69,1 & 0 & 0 & 0 & 0 & 18,5 & $\mathbf{1 0 0}$ \\
& Pil & 0 & 0 & 20,0 & 20,0 & 0 & 0 & 0 & 0 & 60,0 & $\mathbf{1 0 0}$ \\
& Vel & 0 & 0 & 0 & 33,3 & 0 & 0 & 0 & 0 & 66,7 & $\mathbf{1 0 0}$ \\
& Sch & 16,7 & 0 & 0 & 33,3 & 0 & 0 & 0 & 16,7 & 33,3 & $\mathbf{1 0 0}$ \\
& Fle & 0 & 0 & 0 & 0 & 0 & 0 & 0 & 57,1 & 42,9 & $\mathbf{1 0 0}$ \\
& Dal & 0 & 0 & 0 & 19,7 & 0 & 0 & 0 & 0 & 80,3 & $\mathbf{1 0 0}$ \\
\hline
\end{tabular}

A morfologia pode ser analisada separadamente em termos de tamanho e forma e ambos os aspectos indicam diferenças importantes entre os grupos analisados. Em termos de tamanho, verifica-se que as pontas da Gruta do Marinheiro e do Tunas são, de maneira geral, menores em relação a alguns grupos (Alice Boer, Bom Jardim Velho e Dalpiaz). Já os espécimes de Alice Boer tendem a ser, em média, maiores do que os de Tunas, Garivaldino e Dalpiaz (Okumura \& Araujo 2013; 2015b; 2016). Por último, as pontas de Dalpiaz seriam, em média, maiores do que as de Garivaldino. Os resultados relativos ao tamanho apontam para diferenças importantes entre os grupos, onde chama a atenção os grupos Gruta do Marinheiro e Tunas por seu tamanho pequeno, ao passo que Alice Boer se destaca pelo seu tamanho grande.

Em termos de forma, também parece haver diferenças importantes entre alguns dos grupos analisados. É possível caracterizar as pontas de Dalpiaz como sendo pouco reavivadas (ou seja, com corpos alongados) e com pedúnculos curtos e convexos, ao passo que os materiais de Alice Boer são pouco reavivados, com pedúnculos longos cujas bases são convexas ou afiladas. Já os materiais da Gruta do Marinheiro tendem a ser reavivados (corpos curtos) com pedúnculos convexos. As pontas do Tunas apresentam corpos curtos (o que sugere uma grande importância do reavivamento) e pedúnculos côncavos ou bifurcados. Essa diferenciação em termos da morfologia dos pedúnculos apoia a nossa hipótese inicial de que essa parte da ponta estaria menos sujeita a pressões seletivas e seria um potencial marcador de diferenças entre grupos pré-históricos.

De modo geral, pode-se dizer que não há diferenças marcantes entre os grupos do Rio Grande do Sul (Okumura \& Araujo 2013; 2015b; 2016), embora algumas diferenças em termos de tamanho tenham sido observadas entre Dalpiaz e Garivaldino. As diferenças observadas na forma das pontas de Morro da Flecha 1 e os demais grupos gaúchos devem ser pensadas levando-se em conta o pequeno tamanho amostral desse grupo.

Em suma, nossas análises permitem vislumbrar uma série de questões que podem ser atacadas com outros métodos e por outros meios, mas que só puderam ser levantadas pela 
aplicação de uma abordagem morfométrica e estatística. A aparente homogeneidade das pontas foi contestada. É possível perceber variações regionais que sugerem fortemente um maior fluxo de ideias em determinadas áreas, em contraste com fronteiras que separam as formas de outras áreas. Por fim, nossos dados suportam a utilidade do conceito heurístico que propõe a dicotomia entre estilo e função (Dunnell 1978a). Muita confusão reina na literatura sobre esse tópico, e essa confusão emerge do não entendimento de que a dicotomia é teórica, ou seja, de que "estilo" e "função" são conceitos, e não “coisas”. Esperamos que nossos dados possam inspirar novas abordagens e a colocação de novos problemas no âmbito dos estudos de caçadores-coletores no Sudeste e Sul do Brasil.

\section{Agradecimentos}

Agradecemos a Edward Koole pela escavação cuidadosa do material da Gruta do Marinheiro e pela disposição em compartilhar tais materiais para essa pesquisa. Também agradecemos a todos que colaboraram com esta pesquisa através do generoso acesso às coleções, em especial: Márcia Rabelo (MAC/Pains), João Boer (in memoriam), Aparecida Boer, Igor Chmyz (CEPA/UFPR), Adriana Schmidt Dias (UFRGS), Jefferson Dias (Marsul), Sérgio Klamt (CEPA-UNISC) e Pedro Ignácio Schmitz (IAP/Unisinos). Esta pesquisa teve o apoio financeiro do CNPq (MO: 303566/2014-0, 443169/2014-4, 443242/2015-1; AGMA: 302670/2015-7), FAPESP (AGMA: 2013/13794-5) e British Academy - Newton Mobility Grants Scheme (MO: NG140077).

\section{Referências}

Araujo, A.G.M. 2012, Projeto “A Ocupação Paleoíndia no Estado de São Paulo: Uma Abordagem Geoarqueológica” Relatório Final de Atividades (FAPESP). Processo FAPESP no. 2009/54720-9. Março de 2010 - Março de 2012. Museu de Arqueologia e Etnologia, Universidade de São Paulo, São Paulo, 222 p. (in Portuguese) (“The Paleoindian Occupation in São Paulo State: A geoarchaeological approach”)

Bentley, R.A. 2011, 'Style versus Function’ 30 Years On. In: Evolutionary and Interpretive Archaeologies: A Dialogue (Cochrane, E.E. \& Gardner, A., Eds.), Left Coast Press, Walnut Creek, California: p. 83-104. (em inglês) ("Estilo versus função 30 anos depois”)

Bettinger, R.L. \& Eerkens, J.W. 1997, Evolutionary implications of metrical variation in Great Basin projectile points. Archeological Papers of the American Anthropological Association, 7(1): 177-191. (em inglês) ("Implicações evolucionárias da variação métrica em pontas de projéteis da Grande Bacia”) doi:10.1525/ap3a.1997.7.1.177

Binford, L.R. 1986, An Alyawara day: making men's knives and beyond. American Antiquity, 51(3): 547-562. (em inglês) (“Um dia Alyawara: fazendo as facas dos homens e mais") doi:10.2307/281751

Bookstein, F.L. 1991, Morphometric Tools for Landmark Data: Geometry and Biology. Cambridge University Press, Cambridge, 435 p. (em inglês) ("Ferramentas morfométricas para dados de pontos de referências: Geometria e biologia”)

Braun, D.P. 1995, Style, selection, and historicity. In: Style, Society, and Person: Archaeological and Ethnological Perspectives (Interdisciplinary Contributions to Archaeology) (Carr, C. \& Neitzel, J.E., Eds.), Plenum Press, New York: p. 123-141. (em inglês) ("Estilo, seleção e historicidade”) 
Braun, D.P. \& Plog, S. 1982, Evolution of “Tribal” Social Networks: Theory and Prehistoric North American Evidence. American Antiquity, 47: 504-525. (em inglês) ("Evolução das redes sociais "tribais": Teoria e evidência pré-histórica norte americana”) doi: $10.2307 / 280232$

Brochado, J.P. \& Schmitz, P.I. 1973, Aleros y cuevas com petroglifos y industria lítica de la escarpa del Planalto Meridional en Rio Grande do Sul, Brasil. Anales de Arqueologia y Etnologia (Mendoza), 27-28: 39-66. (em espanhol) ("Lajes e cavernas com petroglifos e indústria lítica da escarpa do Planalto Meridional no Rio Grande do Sul, Brasil”)

Childe, G.V. 1951, Social evolution. Watts and Co., London. 184 p. (em inglês) ("Evolução social")

Childs, T.S. 1991, Style, technology, and iron smelting furnaces in Bantu-speaking Africa. Journal of Anthropological Archaeology, 10(4): 332-359. (em inglês) ("Estilo, tecnologia, e fornos de fundição de ferro na África Bantu”) doi:10.1016/02784165(91)90006-J

Chmyz, I., Sganzerla, E.M., Volcov, J.E., Bora, E. \& Ceccon, R.S. 2008, A arqueologia da área da LT 750kV Ivaiporã-Itaberá III, Paraná - São Paulo. Arqueologia, (Número Especial 5), 20(1): 1-305. (in Portuguese) (“Archaeology of LT 750kV Ivaiporã-Itaberá III, Paraná - São Paulo State”)

URL: http://kvasirpublishing.com/journals/arq/article/view/119

Conkey, M.W. 1978, Style and information in cultural evolution: toward a predictive model for the Paleolithic. In: Social Archaeology: Beyond Subsistence and Dating (Redman, C.L., Berman, M.J., Curtin, E.V., Langhorne, W.T., Jr., Versaggi, N.M. \& Wanser, J.C., Eds.), Academic Press, New York: p. 61-85. (em inglês) ("Estilo e informação em evolução cultural: Em busca de um modelo preditivo para o Paleolítico”)

Danzeglocke, U., Jöris, O. \& Weninger, B. (2007). CalPal Online (Version 1.5). Cologne: University of Cologne. URL: http://www.calpal-online.de

David, N. \& Kramer, C. 2001, Ethnoarchaeology in action. Cambridge World Archaeology Series, Cambridge University Press, Cambridge, 508 p. (em inglês) ("Etnoarqueologia em ação”) doi:10.1017/CBO9781316036488

Dias, A.S. 2003, Sistemas de assentamento e estilo tecnológico: Uma proposta interpretativa para a ocupação pré-colonial do alto Vale do Rio dos Sinos, Rio Grande do Sul. Tese de doutorado na Faculdade de Filosofia, Letras e Ciências Humanas, Universidade de São Paulo, São Paulo, 326 p. + anexos. (in Portuguese) ("Settlement systems and technological style: An interpretative proposal for precolonial occupation of upper Sinos River valley, Rio Grande do Sul State”)

Dias, A.S. 2007, Novas perguntas para um velho problema: Escolhas tecnológicas como índices para o estudo de fronteiras e identidades sociais no registro arqueológico. Boletim do Museu Paraense Emílio Goeldi (Ciências Humanas), 2(1): 59-76. (in Portuguese) ("New questions for an old problem: Technological choices as indexes to the study of social boundaries and identities in the archaeological record") doi:10.1590/S1981-81222007000100005

Dias, A.S. 2012, Hunter-gatherer occupation of south Brazilian Atlantic Forest: Paleoenvironment and archaeology. Quaternary International, 256: 12-18. (em inglês) (“Ocupação de caçadores-coletores do floresta atlântica brasileira do sul: Paleoambiente e arqueologia”) doi:10.1016/j.quaint.2011.08.024 
Dias, A.S. \& Hoeltz, S.E. 2010, Indústrias Líticas em Contexto: O Problema Humaitá na Arqueologia Sul Brasileira. Revista de Arqueologia, 23: 40-67. (in Portuguese) ("Lithic industries in context: The Humaitá problem in Southern Brazilian Archaeology”) URL: http://www.revista.sabnet.com.br/revista/index.php/SAB/article/view/299

Dunnell, R.C. 1978a, Style and function: A fundamental dichotomy. American Antiquity, 43: 192-202. (em inglês) ("Estilo e função: Uma dicotomia fundamental”) doi:10.2307/279244

Dunnell, R.C. 1978b, Archaeological potential of anthropological and scientific models of function. In: Archaeological Essays in Honor of Irving Benjamin Rouse (Dunnell, R.C., Ed.), Mouton Publishers, The Hague: p. 41-73. (em inglês) ("Potencial arqueológico dos modelos de função baseados na antropologia e na ciência”)

Dunnell, R.C. 2001, Foreword. In: Style and function: Conceptual issues in evolutionary archaeology (Hurt, T.D. \& Rakita, G.F.M., Eds.), Bergin \& Harvey, Westport: p. xiiixxix. (em inglês) ("Prefácio”)

Gosselain, O.P. 1992, Technology and style: Potters and pottery among Bafia of Cameroon. Man, 27: 559-586. (em inglês) ("Tecnologia e estilo: Oleiros e cerâmicas entre os Bafia dos Camarões”) doi:10.2307/2803929

Griffin, P.B. 1997, Technology and Variation in Arrow Design among the Agta of Northeastern Luzon. In: Projectile Technology (Knecht, H., Ed.), Plenum Press, New York: p. 267-286. (em inglês) ("Tecnologia e variação no design de flechas entre os Agta do nordeste de Luzon”)

Hegmon, M. 1992, Archaeological research on style. Annual Review of Anthropology, 21: 517-536. (em inglês) ("Pesquisa arqueológica sobre estilo”) doi:10.1146/annurev.an.21.100192.002505

Hegmon, M. 1998, Technology, style and social practices: Archaeological approaches. In: The archaeology of social boundaries (Stark, M., Ed.), Smithsonian Institution Press, Washington: p. 264-279. (em inglês) ("Tecnologia, estilo e práticas sociais: Abordagens arqueológicas”)

Hilbert, K. 1991, Aspectos de la Arqueología en el Uruguay. Ava Materialien Vol. 44. Verlang Philipp von Zabern, Mainz Am Rhein, 137 p. (em espanhol) (Aspectos da Arqueologia no Uruguai) (in Portuguese) (“Aspects of Archaeology in Uruguay”)

Hilbert, K. 1994, Caçadores coletores pré-históricos no sul do Brasil: Um projeto para a redefinição das tradições líticas Umbu e Humaitá. In: Negros e índios: Literatura e história (Flores, M., Ed.), Editora da Pontifícia Universidade Católica do Rio Grande do Sul (EdiPUCRS), Porto Alegre: p. 9-24. (in Portuguese) ("Prehistoric hunter-gatherers in the South of Brazil: A Project for redefining the Umbu and Humaitá lithic industries")

Hitchcock, R. \& Bleed, P. 1997, Each according to need and fashion: Spear and arrow use among San hunters of the Kalahari. In: Projectile Technology (Knecht, H., Ed.), New York and London: p. 345-368. (em inglês) ("Cada um de acordo com a necessidade e a moda: Uso de lanças e flechas entre os caçadores San do Kalahari”)

Hodder, I. 1982, Symbols in action: Ethnoarchaeological studies of material culture. Cambridge University Press, Cambridge, 244 p. (em inglês) ("Símbolos em Ação: Estudos etnoarqueológicos de cultura material”) 
Hurt, T.D. \& Rakita, G.F.M. 2001, Style and function: Conceptual issues in evolutionary archaeology. Bergin \& Harvey, Westport, 240 p. (em inglês) ("Estilo e função: Problemas conceituais em arqueologia evolutiva”)

Kendall, D.G. 1977, The diffusion of shape. Advances in Applied Probability, 9(3): 428-430. (em inglês) (“A difusão do contorno”) doi:10.2307/1426091

Koole, E.K.M. 2007, Pré-história da província Cárstica do Alto São Francisco, Minas Gerais: A indústria lítica dos caçadores-coletores arcaicos. Dissertação de mestrado no Museu de Arqueologia e Etnologia, Universidade de São Paulo, São Paulo, 139 p. (in Portuguese) ("Prehistory of Upper São Francisco karst province, Minas Gerais State: The archaic hunter gatherer lithics industries”)

Koole, E.K.M. 2014, Entre as tradições planálticas e meridionais: Caracterização arqueológica dos grupos caçadores coletores a partir da análise de sete elementos e suas implicações para a ocupação pré-cerâmica da Região Cárstica do Alto São Francisco, Minas Gerais, Brasil: Cronologia, tecnologia lítica, subsistência (fauna), sepultamentos, mobilidade, uso do espaço em abrigos naturais e arte rupestre. Tese de doutorado no Museu de Arqueologia e Etnologia, Universidade de São Paulo, São Paulo, 564 p. (in Portuguese) ("Between plateau and meridional traditions: archaeological characterization of hunter-gatherer groups from seven elements analysis and their implications to pre-pottery occupations of de Upper São Francisco karstic region, Minas Gerais State, Brazil: Chronology, lithic technology, subsistence (faunal remains) burials, mobility, space use in rockshelters and rock art”)

Larick, R. 1985, Spears, style, and time among Maa-speaking pastoralists. Journal of Anthropological Archaeology, 4(3): 206-220. (em inglês) ("Lanças, estilo e tempo entre os pastoralistas falantes de Maa”) doi:10.1016/0278-4165(85)90003-0

Lechtman, H.N. 1977, Style and Technology: Some Early Thoughts. In: Material Culture: Styles,Organization, and Dynamics of Technology (Lechtman, H.N. \& Merrill, R.S., Eds.), West Publishing, St. Paul: p. 3-20. (em inglês) ("Estilo e tecnologia: Alguns pensamentos iniciais”)

Lechtman, H.N. \& Steinberg, A. 1979, The History of Technology: An Anthropological Perspective. In: History and Philosophy of Technology (Bugliarello, G. \& Doner, D.B., Eds.), University of Illinois Press, Urbana: p. 135-160. (em inglês) (“A história da tecnologia: Uma perspectiva antropológica”)

Lima, A.P.S. 2005, Análise dos processos formativos do Sítio Capelinha - estabelecimento de um contexto microrregional, Dissertação de mestrado. Museu de Arqueologia e Etnologia, Universidade de São Paulo, São Paulo, 136 p. (in Portuguese) (“The Capelinha site formation process analysis: Establishing a microrregional context”)

Lipo, C.P. \& Madsen, M. 2001, Neutrality, “style”, and drift: Building methods for studying cultural transmission in the archaeological record. In: Style and function: Conceptual issues in evolutionary archaeology (Hurt, T.D. \& Rakita, G.F.M., Eds.), Bergin \& Harvey, Westport, Minnesota: p. 91-118. (em inglês) (“Neutralidade, "estilo”, e deriva: Construindo métodos para estudos de transmissão cultural no registro arqueológico”)

Lipo, C.P., Madsen, M.E., Dunnell, R.C. \& Hunt, T. 1997, Population structure, cultural transmission, and frequency seriation. Journal of Anthropological Archaeology, 16(4): 301-333. (em inglês) ("Estrutura populacional, transmissão cultural e seriação de frequência”) doi:10.1006/jaar.1997.0314 
Lipo, C.P., Hunt, T.L. \& Hundtoft, B. 2010, Stylistic variability of stemmed obsidian tools (mata'a), frequency seriation, and the scale of social interaction on Rapa Nui (Easter Island). Journal of Archaeological Science, 37(10): 2551-2561. (em inglês) ("Variabilidade estilística de ferramentas pedunculadas de obsidiana - mata’a - seriação de frequência e a escala de interação social em Rapa Nui, Ilha de Páscoa”) doi:10.1016/j.jas.2010.05.015

McElreath, R., Boyd, R. \& Richerson, P.J. 2003, Shared norms can lead to the evolution of ethnic markers. Current Anthropology, 44(1): 122-130. (em inglês) ("Normas compartilhadas podem levar à evolução de marcadores étnicos”) doi:10.1086/345689

Milder, S.E.S. 1999, Caçadores coletores: A problemática arqueológica e ambiental sobre os primeiros povoadores do Rio Grande do Sul. Revista do CEPA (Santa Cruz do Sul), 23: 7-56. (in Portuguese) ("Hunter gatherers: The archaeological and environmental problem about the first people in Rio Grande do Sul State”)

Miller, E.T. 1967, Pesquisas Arqueológicas Efetuadas no Nordeste do Rio Grande do Sul. In: Programa Nacional de Pesquisas Arqueológicas: Resultados Preliminares do Primeiro Ano (1965-1966) (Simões, M., Ed.), Publicações Avulsas do Museu Paranaense Emílio Goeldi Vol. 6, Museu Paranaense Emílio Goeldi, Belém: p. 15-38. (in Portuguese) (“Archaeological research carried out in northeastern Rio Grande do Sul state”)

Miller, E.T. 1974, Pesquisas Arqueológicas em Abrigos-sob-Rocha no Nordeste do Rio Grande do Sul. In: Programa Nacional de Pesquisas Arqueológicas: Resultados Preliminares do Quinto Ano (1969-1970) (Simões, M., Ed.), Publicações Avulsas do Museu Paranaense Emílio Goeldi Vol. 26, Museu Paranaense Emílio Goeldi, Belém: p. 11-24. (in Portuguese) (“Archaeological research in rockhelters in northeastern Rio Grande do Sul state”)

Neiman, F.D. 1995, Stylistic variation in evolutionary perspective: Inferences from decorative diversity and interassemblage distance in Illinois Woodland ceramic assemblages. American Antiquity, 60(1): 7-36. (em inglês) ("Variação estilística em perspectiva evolutiva: Inferências da diversidade decorativa e distância intercoleções em coleções cerâmicas Woodland de Illinois”) doi:10.2307/282074

Noelli, F.S. 2000, A ocupação humana na região sul do Brasil: Arqueologia, debates e perspectivas. Revista USP, 44: 218-269. (in Portuguese) ("The human occupation in the south of Brazil: Archaeology, debates and perspectives”) doi:10.11606/issn.23169036.v0i44p218-269

O’Brien, M.J. \& Leonard, R.D. 2001, Style and function: An introduction. In: Style and function: Conceptual issues in evolutionary archaeology (Hurt, T.D. \& Rakita, G.F.M., Eds.), Bergin \& Harvey, Westport: p. 1-23. (em inglês) (“Estilo e função: uma introdução”)

Okumura, M. \& Araujo, A.G.M. 2013, Pontas bifaciais no Brasil Meridional: Caracterização estatística das formas e suas implicações culturais. Revista do Museu de Arqueologia e Etnologia, 23: 111-127. (in Portuguese) (“Bifacial points in Meridional Brazil: Statistical characterization of forms and their cultural implications”) URL: http://www.producao.usp.br/handle/BDPI/47557

Okumura, M. \& Araujo, A.G.M. 2014, Long-term cultural stability in hunter-gatherers: A case study using traditional and geometric morphometric analysis of lithic stemmed bifacial points from Southern Brazil. Journal of Archaeological Science, 45: 59-71. (em inglês) ("Estabilidade cultural a longo prazo em caçadores-coletores: Um caso de estudo 
usando morfometria geométrica e tradicional de pontas líticas pedunculadas do sul do Brasil”) doi:10.1016/j.jas.2014.02.009

Okumura, M. \& Araujo, A.G.M. 2015a, Contributions to the Dart versus Arrow Debate: New Data from Holocene Projectile Points from Southeastern and Southern Brazil. Anais da Academia Brasileira de Ciências, 87(4): 2349-2373. (em inglês) (“Contribuições ao debate dardo versus flecha: Novos dados de pontas de projéteis holocências do sudeste e sul do Brasil”) doi:10.1590/0001-3765201520140625

Okumura, M. \& Araujo, A.G.M. 2015b, Desconstruindo o que nunca foi construído: Pontas bifaciais ‘Umbu’ do Sul e Sudeste do Brasil. Revista do Museu de Arqueologia e Etnologia, Suplemento 20: 77-82. (in Portuguese) ("Deconstructing what was never constructed: Umbu bifacial points in South and Southeast Brazil”)

URL: http://www.producao.usp.br/handle/BDPI/49254

Okumura, M. \& Araujo, A.G.M. 2016, The Southern Divide: Testing morphological differences among bifacial points from southern and southeastern Brazil using geometric morphometrics. Journal of Lithic Studies, 3(1): 107-131. (em inglês) (“O sul dividido: Testando diferenças morfológicas entre pontas bifaciais do sul e sudeste do Brasil usando morfometria geométrica”) doi:10.2218/jls.v3i1.1379

Prous, A. 1991, Arqueologia brasileira. Universidade de Brasília, Brasília, 605 p. (in Portuguese) ("Brazilian archaeology")

Queiroz, A.N. 2004, Etude des vertébrés du site RS-TQ-58, Montenegro, RS, Brésil: Aspects archéozoologiques et taphonomiques. In: Zooarchaeology of South America (Goñalons, G.L.M., Ed.), British Archaeological Reports, International Series Vol. 1298, Archaeopress, Oxford: p. 153-176. (em francês) ("Estudo dos vertebrados do sítio RSTQ-58, Montenegro, RS, Brasil: Aspectos arqueozoológicos e tafonômicos”) (in French) ("Study of the vertebrates of the RS-TQ-58 site, Montenegro, RS, Brazil: Archaeozoological and taphonomic aspects”)

Ribeiro, P.A.M., Klamt, S.C., Buchaim, J.J.S. \& Ribeiro, C.T. 1989, Levantamentos arqueológicos na encosta do planalto entre o vale dos rios Taquari e Caí, RS, Brasil. Revista do CEPA (Santa Cruz do Sul), 16(19): 49-89. (in Portuguese) (“Archaeologcial surveys in the hillside of Taquari and Caí rivers valley, RS, Brazil”)

Ribeiro, P.A.M. \& Ribeiro, C.T. 1999, Escavações Arqueológicas no Sítio RS-TQ-58: Montenegro, RS, Brasil. Série Documento (Editora da FURG), 10: 1-86. (in Portuguese) (“Archaeological excavations in the RS-TQ-58 site: Montenegro, RS, Brasil”)

Rick, J. 1996, Projectile points, style, and social process in the preceramic of central Peru. In: Stone Tools: Theoretical Insights into Human Prehistory (Odell, G.H., Ed.), Plenum Press, New York: p. 245-278. (em inglês) ("Pontas de projéteis, estilo e processo social no centro do Peru pré-cerâmico”)

Roe, P.G. 1995, Style, society, myth, and structure. In: Style, society and person: Archaeological and ethnological perspectives (Carr, C. \& Neitzel, J.E., Eds.), Plenum Press, New York: p. 27-76. (em inglês) (“Estilo, sociedade, mito e estrutura”)

Rohlf, F.J. (2017). TPS Series Software. New York: Department of Ecology and Evolution, State University of New York Stony Brook, Stony Brook.

URL: http://life.bio.sunysb.edu/morph/soft-tps.html 
Rosa, A.O. 2009, Análise Zooarqueológica do Sítio Garivaldino (RS-TQ-58), Município de Montenegro, RS. Pesquisas Antropologia, 67: 133-171. (in Portuguese)

(“Zooarchaeological analysis of the Garivaldino Site (RS-TQ-58), Montenegro municipality, RS”)

URL: http://www.anchietano.unisinos.br/publicacoes/antropologia/antropologia67/Rosa .pdf

Sackett, J.R. 1985, Style and ethnicity in the Kalahari: A reply to Wiessner. American Antiquity, 50: 154-159. (em inglês) ("Estilo e etnicidade no Kalahari: Uma réplica a Wiessner”) doi:10.2307/280642

Sackett, J.R. 1986, Style, function, and assemblage variability: A reply to Binford. American Antiquity, 51(3): 628-634. (em inglês) ("Estilo, função e variabilidade em coleções: Uma réplica a Binford”) doi:10.2307/281759

Schmitz, P.I. 1978, Industrias líticas en el sur de Brasil. Estudos Leopoldenses, 14(47): 103132. (em espanhol) ("Indústrias líticas no sul do Brasil”) (in Spanish) (“Lithic industries in the south of Brasil”)

Schmitz, P.I. 1999, A questão do Paleoíndio. In: Pré-História da Terra Brasilis (Tenório, M.C., Ed.), Editora UFRJ, Rio de Janeiro: p. 55-59. (in Portuguese) ("The palaeoindian question”)

Schmitz, P.I. 2006, Sítio Pedro Fridolino Schmitz, Bom Princípio, RS. Número do sítio RS 217. In: Anais do V encontro do Núcleo Regional Sul da Sociedade de Arqueologia Brasileira; SAB/Sul, Rio Grande, RS 20 a 23 de novembro. Sociedade de Arqueologia Brasileira \& Fundação Universidade Federal de Rio Grande, Rio Grande: 23 p. (in Portuguese) (“The Pedro Fridolino Schmitz site, Bom Princípio, RS”)

URL: http://www.anchietano.unisinos.br/sabsul/V\%20\%20SABSul/simposio/atlantica/9.pdf

Schmitz, P.I., Barbosa, A.S. \& Ribeiro, P.M. 1980, Temas de Arqueologia Brasileira 1 Paleoindio. Vol. 5. Anuário de Divulgação Científica. Universidade Católica de Goiás, Goiânia, 99 p. (in Portuguese) (“Brazilian Archaeology Themes 1 - Paleoindian”)

Shennan, S.J. 1989, Introduction: Archaeological approaches to cultural identity. In: Archaeological Approaches to cultural identity (Shennan, S.J., Ed.), Unwin Hyman, London: p. 1-32. (em inglês) ("Introdução: Abordagens arqueológicas para o estudo da identidade cultural”)

Shennan, S.J. \& Wilkinson, J.R. 2001, Ceramic style change and neutral evolution: A case study from Neolithic Europe. American Antiquity, 66(4): 577-593. (em inglês) ("Mudanças de estilo cerâmico e evolução neutra: Um caso de estudo do Neolítico Europeu”) doi:10.2307/2694174

Stuiver, M., Reimer, P.J., Bard, E., Beck, J.W., Burr, G.S., Hughen, K.A., Kromer, B., McCormac, G., van der Plicht, J. \& Spurk, M. 2006, INTCAL98 radiocarbon age calibration, 24,000-0 cal BP. Radiocarbon, 40(3): 1041-1083. URL: https://journals.uair.arizona.edu/index.php/radiocarbon/article/view/3781

Wiessner, P. 1983, Style and social information in Kalahari San projectile points. American Antiquity, 48(2): 253-276. (em inglês) ("Estilo e informação social nas pontas de projéteis Kalahari San”) doi:10.2307/280450

Wiessner, P. 1984, Reconsidering the behavioral basis for style: A case study among the Kalahari San. Journal of Anthropological Archaeology, 3(3): 190-234. (em inglês) 
("Reconsiderando a base comportamental para estilo: um caso de estudo entre o Kalahari San”) doi:10.1016/0278-4165(84)90002-3

Wobst, H.M. 1977, Stylistic behavior and information exchange. In: For the Director: Research essays in honor of James B. Griffin (Cleland, C.E., Ed.), University of Michigan Museum of Anthropology, Ann Arbor: p. 317-342. (em inglês)

(“Comportamento estilístico e troca de informação")

Zelditch, M.L., Swiderski, D.L., Sheets, D.H. \& Fink, W.L. 2004, Geometric Morphometrics for Biologists: A Primer. Elsevier, Academic Press, New York, 443 p. (em inglês) ("Morfometria geométrica para biólogos: Uma introdução”) 


\title{
Southern and southeastern boundaries: A geometric morphometric analysis of bifacial points from Minas Gerais, São Paulo, Paraná, and Rio Grande do Sul (Brazil)
}

\author{
Mercedes Okumura ${ }^{1}$, Astolfo G. M. Araujo ${ }^{2}$ \\ 1. PPGArq, Department of Anthropology, National Museum, Federal University of Rio de Janeiro, Brazil. \\ Email: mercedes@mn.ufrj.br \\ 2. Institute for Advanced Studies and Museum of Archaeology and Ethnology, University of São Paulo, Brazil. \\ Email: astwolfo@usp.br
}

\begin{abstract}
:
Style and function in archaeological or ethnographic artifacts have been studied by a myriad of researchers through the most diverse theoretical approaches. In this context, the study of projectile points has been particularly useful in generating a greater knowledge about these artifacts as markers of identity, reflecting potential boundaries among groups. This work aims to explore the differences in the morphology of Holocene bifacial points from Minas Gerais, São Paulo, Paraná and Rio Grande do Sul in light of the concepts of style and function given by Evolutionary Archaeology. 248 points were analyzed by two dimensional geometric morphometrics. Although our analysis involves geometric morphometrics data of the complete point (blade and stem), the shape of the stem was considered to be of particular importance. According to the theoretical expectations and the heuristic models of style and function proposed by Dunnell (1978a), differences in the shape of the stem of the points can be considered a stylistic resource whose aspect and differential replication among groups was most likely due to stochastic processes. Thus, our theoretical expectation is that the shape of the stem reflects the choices of the groups and, ultimately, is a reflection of potential ancient cultural boundaries. Our results point to the presence of important differences in the size and shape of the points from Minas Gerais, São Paulo, and Paraná in relation to the points from Rio Grande do Sul. In terms of size, points from Minas Gerais and Paraná are smaller than most groups, while points from São Paulo tend to be bigger. Moreover, our analysis shows that there are important differences in terms of both blade shape (mostly related to resharpening) and stem shape. Points from the Dalpiaz site (Rio Grande do Sul state) and São Paulo state present very low resharpening, resulting in elongated blades. However, the stem morphology of these two samples is quite different: at the Dalpiaz site, stems are short and convex, while in São Paulo points, stems are long and convex. Minas Gerais and Paraná samples present short blades, but striking differences in the stem morphology (the former can be characterized as convex and the later as concave shape). The fact that important differences can be observed in the stem morphology, shows the benefit of the application of the heuristic dichotomy between style and function (sensu Dunnell 1978a) to better understand the presence of potential past cultural boundaries. It was possible to observe regional variations in the morphology of the points that strongly suggest a greater flux of ideas in certain areas, in comparison to other areas that seem more isolated. Finally, it is possible to suggest that, in general, materials from Rio Grande do Sul seem to be quite homogeneous, although important differences were observed in terms of size (especially between materials from the Dalpiaz and Garivaldino sites). The small sample size of most analyzed groups from Rio Grande do Sul might be a factor that should be taken into account in this discussion and future analysis including a greater sample size should be done in order to better understand such diversity.
\end{abstract}

Keywords: Umbu tradition; lithic points; style and function; geometric morphometrics; identity 